Accepted manuscript version of: Leduc, Guillaume. "A European Option Binomial Scheme General First Order Error Formula." ANZIAM Journal 54, no. 4 (August, 2013): 248-272. doi: 10.1017/S1446181113000254.

(C)2013 Australian Mathematical Society

\title{
A EUROPEAN OPTION GENERAL FIRST-ORDER ERROR FORMULA
}

\author{
GUILLAUME LEDUC
}

\begin{abstract}
We study the value of European security derivatives in the Black-Scholes model, when the underlying asset $\xi$ is approximated by random walks $\xi^{(n)}$. We obtain an explicit error formula, up to a term of order $\mathcal{O}\left(n^{-\frac{3}{2}}\right)$, which is valid for general approximating schemes and general payoff functions. We show how this error formula can be used to find random walks $\xi^{(n)}$, for which option values converge at a speed of $\mathcal{O}\left(n^{-\frac{3}{2}}\right)$.
\end{abstract}

\section{INTRODUCTION}

1.1. Motivation. The problem of describing and controlling the error for options evaluated under random walk approximations $\left\{\xi^{(n)}\right\}$ of a geometric Brownian motion $\xi$ has attracted the attention of many researchers (see, for instance, [1], [2], [3], [4], [5], [6], [7], [8], [9], [10], [11], [12], [13], [14], $[15],[16],[17],[18],[19],[20],[21],[22],[23],[24],[25])$. Knowledge and control of the error resulting from evaluating options through random walk approximations is of immediate interest, as random walks are broadly used to price them. An explicit error formula, up to an error term of order $n^{-\alpha}$, for some $\alpha>0$, has allowed an "acceleration" of the speed of convergence to an order of $n^{-\alpha}$ in [13] and [11]. In a broader context, such error formulae contribute to the understanding of how small modelling errors affect option prices, which is intimately related to the important question of option price robustness.

It is common practice to approximate a real valued function $f(x)$ by its Taylor expansion, which, for $f$ sufficiently regular, is given around $a$ by

$$
f(x)=\sum_{k=0}^{N} \frac{f^{(k)}(a)}{k !}(x-a)^{k}+\int_{a}^{x} \frac{f^{(k+1)}(t)}{k !}(x-t)^{k} d t .
$$

The first-order term $f^{(1)}(a)$ provides a measure of the sensitivity of $f$ to small changes of its parameter $x$ around $a$.

In the case of an option, its value $v$ depends on the distribution of the underlying asset $\xi$, and small random/unknown changes in the distribution

Date: 1 May 2013.

1991 Mathematics Subject Classification. Primary 91G20; Secondary 91G60.

Key words and phrases. European options, approximation scheme, error formula, Black-Scholes. 
of $\xi$ induce a "modelling error" in the pricing. One would like to have an analogue to Taylor's expansion for the value $v(\xi)$ of an option, seen as a function of the distribution of the underlying asset $\xi$, that would help to price and perhaps hedge the modelling error.

In the case of a binomial approximation scheme $\left\{\xi^{(n)}\right\}$ of the underlying asset $\xi$ in the Black-Scholes model, such an analogue to Taylor's expansion takes the form

$$
v(\xi)=v\left(\xi^{(n)}\right)+\sum_{k=1}^{N} v_{k}\left(\xi^{(n)}\right) n^{-\frac{k}{2}}+\mathcal{O}\left(n^{-\frac{N+1}{2}}\right) .
$$

If $N=2$ then $n^{-\frac{N}{2}}=n^{-1}$, and explicit formulae for the coefficients $v_{1}\left(\xi^{(n)}\right)$ and $v_{2}\left(\xi^{(n)}\right)$ provide what we call a first-order error formula. Walsh [25] gives such a formula for general piecewise $C^{(2)}$ payoffs, but only in the specific case where the binomial scheme is the Cox, Ross and Rubinstein scheme, applied to the discounted process. Diener and Diener [4] provide a first-order error formula for general binomial schemes, but only in the specific case where the payoff is a call option. In [5] they obtain this firstorder error formula for digital options. The present paper fills the obvious gap: we obtain a first error formula which is valid for both general payoffs and for general binomial scheme approximations.

Chang and Palmer [2] showed how knowledge of a first-order error formula can be used to obtain schemes for which the error is smooth, that is for which the error has the form $c n^{-1}+o\left(n^{-1}\right)$ for some constant $c$. Korn and Müller [13], developed an optimization procedure to minimize the absolute value of this $c$. We will show here how, using the error formula obtained in this paper, a slight modification of Korn and Müller's [13] optimization procedure allows one to reach "accelerations" of the convergence to an order of $\mathcal{O}\left(n^{-1.5}\right)$.

An interesting feature of our paper is that, when the payoff of the option is continuously differentiable, our error formula remains valid for non-binomial scheme approximations $\left\{\xi^{(n)}\right\}$, as long as $\xi_{\frac{T}{n}}^{(n)}$ satisfy the moment conditions $\mathrm{P} 1-\mathrm{P} 5$ given in section 6 . Our error formula is derived from a localization of the error and an expansion of the local errors.

1.2. Main result. Throughout this paper we assume that $r>0$ is the (constant) risk free rate and that $\xi=\left(\xi, \mathcal{F}, E_{x}\right)$ is a geometric Brownian motion with volatility $\sigma$ and drift $r$ under risk neutral probability. Here $\mathcal{F}$ is the usual filtration and $E_{x}$ denotes the expectation when $\xi_{0}=x$.

For all practical purposes, traders are interested in payoff functions which are piecewise smooth. We consider here payoffs $h$ which are piecewise $C^{(3)}$ and for which

$$
x^{\ell}\left|h^{(\ell)}(x)\right| \leq Q\left(1+x^{p}\right) \text { for } \ell=0, \ldots, 3 \text { and every } x \geq 0,
$$


for some integer $p \geq 1$ and some real number $Q$. By piecewise $C^{(3)}$, we mean that there exists a partition $0<K_{1}<\ldots<\mathrm{K}_{N}<\infty$ of $[0, \infty)$ and $N+1$ functions $h_{0}, \ldots, h_{N} \in C^{(3)}$ such that

$$
h=h_{0} 1_{\left[0, K_{1}\right)}+h_{1} 1_{\left[K_{1}, K_{2}\right)}+\ldots+h_{N} 1_{\left[K_{N}, \infty\right)} .
$$

We denote this class of payoffs by $\mathcal{K}_{p}^{(3)}$. We put a norm \|\|$_{p}^{(3)}$ on $\mathcal{K}_{p}^{(3)}$ equal to the smallest value of $Q$ for which (1.1) holds. For any integer $m \geq 0$, we define $\mathcal{K}_{p}^{(m)}$ and \|\|$_{p}^{(m)}$ analogously.

We want to provide a first-order error formula, when $\xi$ is approximated by binomial schemes $\left\{\xi^{(n)}\right\}_{n=1}^{\infty}$ where $\xi^{(n)}$ is a random walk which, at every positive time $t$ in $\frac{T}{n} \mathbb{N}$, has a probability $p_{n}$ of jumping from its current state $\xi_{t}^{(n)}$ to the state $\xi_{t}^{(n)} u_{n}$, and a probability $1-p_{n}$ of jumping to the state $\xi_{t}^{(n)} d_{n}$. Risk neutrality requires that $p_{n}$ be equal to

$$
p_{n}:=\frac{\exp \left(r \frac{T}{n}\right)-d_{n}}{u_{n}-d_{n}},
$$

with

$$
\begin{aligned}
& u_{n}:=\exp \left(\sigma \sqrt{\frac{T}{n}}+\lambda \sigma^{2} \frac{T}{n}+\mu_{n} \frac{2 \sigma}{T}\left(\frac{T}{n}\right)^{\frac{3}{2}}\right), \\
& d_{n}:=\exp \left(-\sigma \sqrt{\frac{T}{n}}+\lambda \sigma^{2} \frac{T}{n}+\mu_{n} \frac{2 \sigma}{T}\left(\frac{T}{n}\right)^{\frac{3}{2}}\right),
\end{aligned}
$$

where $\left|\mu_{n}\right| \leq \mathcal{L}$ for some $\mathcal{L}$, one gets fairly general binomial schemes, analogous to those considered in [2] and [13]. We will refer to these schemes as flexible CRR schemes. Because we always assume that $\xi_{0}^{(n)}=\xi_{0}, E_{x}$ also denotes the expectation when $\xi_{0}^{(n)}=x$.

Now if $h$ belongs to $\mathcal{K}_{p}^{(3)}$, then $h$ can be split into a linear combination of digital options and call options, plus a function which is continuously differentiable and in $\mathcal{K}_{p}^{(3)}$. Indeed it is easy to see that

$$
h(x)=g(x)+\sum_{\ell=1}^{N} \Delta h\left(\mathrm{~K}_{\ell}\right) 1_{\left[\mathrm{K}_{\ell}, \infty\right)}(x)+\sum_{\ell=1}^{N} \Delta h^{\prime}\left(\mathrm{K}_{\ell}\right) \max \left(x-\mathrm{K}_{\ell}, 0\right),
$$

where $g$ is $C^{(1)}$ and belongs to $\mathcal{K}_{p}^{(3)}$. Since error formulae for digital and call options are already known, thanks to [4], [5] and [2], the contribution of this paper is to find the error formula for the $C^{(1)}$ part of $h$. For the sake of simplicity, we will restrict our exposition to continuous payoff functions $h$. Given that $\xi_{0}=x$, we denote by $\operatorname{Err}_{T}^{n}(h)(x)$ the error, under the BlackScholes model, resulting from pricing with a flexible CRR scheme $\left\{\xi_{T}^{(n)}\right\}$ a European option with payoff $h$ and maturity $T$. In other words

$$
\operatorname{Err}_{T}^{n}(h)(x):=e^{-r T} E_{x}(h(\xi))-e^{-r T} E_{x}\left(h\left(\xi_{T}^{(n)}\right)\right) .
$$


Let $\mathcal{C}_{K}(z)=\max (z-K, 0)$ denote the payoff of a European call option with strike $K$, and set $\mathfrak{d}_{1}=\frac{\left(\ln \left(\frac{x}{K}\right)+\left(r+\frac{1}{2} \sigma^{2}\right) T\right)}{\sigma \sqrt{T}}$, and $\mathfrak{d}_{2}=\mathfrak{d}_{1}-\sigma \sqrt{T}$. Let also $\operatorname{frac}(z)$ be the fractional part of $z$, with $\operatorname{frac}(z):=-\operatorname{frac}(|z|)$, when $z<0$. The following is due to [4] and [2].

Theorem 1 (Call Option First Order Error Formula). Let $\left\{\xi^{(n)}\right\}$ be a flexible CRR scheme. For every $x>0$, the error $\operatorname{Err}_{T}^{n}\left(\mathcal{C}_{K}\right)(x)$ satisfies

$$
\operatorname{Err}_{T}^{n}\left(\mathcal{C}_{K}\right)(x)=\Lambda_{T}^{n}(K, x)+\mathcal{O}\left(n^{-1.5}\right)
$$

where $\Lambda_{T}^{n}:=\Lambda_{T}^{n}(K, x)$ is given by

$$
\begin{aligned}
\Lambda_{T}^{n} & =\frac{x e^{-0.5 \mathfrak{d}_{1}^{2}}}{24 \sigma \sqrt{2 \pi T}}(A+B), \\
A & =\sigma^{2} T\left(6+\mathfrak{d}_{1}^{2}+\mathfrak{d}_{2}^{2}\right)+12 T^{2}\left(r-\lambda \sigma^{2}\right)^{2}-4 T\left(\mathfrak{d}_{1}^{2}-\mathfrak{d}_{2}^{2}\right)\left(r-\lambda \sigma^{2}\right), \\
B & =48 \sigma^{2} T \mathfrak{f}^{(n)}\left(1+\mathfrak{f}^{(n)}\right),
\end{aligned}
$$

$$
\mathfrak{f}^{(n)}=\operatorname{frac}\left(\frac{1}{2}\left(\ln x-\ln K-\sigma \sqrt{T} \sqrt{n}+\lambda \sigma^{2} T\right) \frac{\sqrt{n}}{\sigma \sqrt{T}}+\mu_{n}\right) .
$$

The following theorem is the main result of this paper. Given a continuous payoff $h$ in $\mathcal{K}_{p}^{(3)}$, it provides a formula for the error $\operatorname{Err}_{T}^{n}(h)(x)$.

Theorem 2 (General First Order Error Formula). Let $\left\{\xi^{(n)}\right\}$ be a flexible CRR scheme and let $p \geq 1$. For every continuous $h$ in $\mathcal{K}_{p}^{(3)}$, if $0<\mathrm{K}_{1}<$ $\ldots<\mathrm{K}_{N}<\infty$ defines a partition of $[0, \infty)$, for which $h$ is $C^{(1)}$ on the corresponding closed subintervals, then for every $x \geq 0$,

$$
\operatorname{Err}_{T}^{n}(h)(x)=\frac{\Upsilon_{T}(h, x)+\sum_{\ell=1}^{N} \Delta h^{\prime}\left(\mathrm{K}_{\ell}\right) \Lambda_{T}^{n}\left(\mathrm{~K}_{\ell}, x\right)}{n}+\mathcal{O}\left(n^{-1.5}\right)
$$

where

$$
\begin{aligned}
\Upsilon_{T}(h, x) & =\left(\frac{1}{2} \Delta_{2}-\frac{1}{3} \Delta_{3}+\frac{1}{4} \Delta_{4}\right) e^{-r T} E_{x}\left(\xi_{T}^{2} h^{\prime \prime}\left(\xi_{T}\right)\right) \\
& +\frac{1}{24} \frac{4 \Delta_{3}-5 \Delta_{4}}{\sigma \sqrt{T}} e^{-r T} E_{x}\left(\xi_{T}^{2} h^{\prime \prime}\left(\xi_{T}\right) \eta_{T}\left(\frac{\xi_{T}}{x}\right)\right) \\
& +\frac{1}{24} \frac{\Delta_{4}}{T \sigma^{2}} e^{-r T} E_{x}\left(\xi_{T}^{2} h^{\prime \prime}\left(\xi_{T}\right)\left(\eta_{T}^{2}\left(\frac{\xi_{T}}{x}\right)-1\right)\right),
\end{aligned}
$$


and

$$
\begin{aligned}
\eta_{T}(z) & =\frac{\ln (z)-\left(r-\frac{1}{2} \sigma^{2}\right) T}{\sqrt{T} \sigma} \\
\Delta_{2} & =-\sigma^{4} T^{2} \lambda+\lambda^{2} \sigma^{4} T^{2}+r^{2} T^{2}+r T^{2} \sigma^{2}+\frac{5}{12} \sigma^{4} T^{2}-2 T^{2} r \sigma^{2} \lambda \\
\Delta_{3} & =2 r T^{2} \sigma^{2}-2 \sigma^{4} T^{2} \lambda+2 \sigma^{4} T^{2} \\
\Delta_{4} & =2 \sigma^{4} T^{2}
\end{aligned}
$$

Remark 1. In Theorem 2, if additionally $h$ is $C^{(1)}$, then the error formula (1.4) remains valid for any approximation scheme satisfying properties P1$P 5$ given in section 6 . This is due to the fact that only these properties are used in the proofs. They boil down to moment conditions, and therefore our error formula remains valid under these broad assumptions on the moments of the single step random walk jumps $\xi_{\frac{T}{n}}^{(n)}$.

Let $\left\{\xi^{(n)}\right\}$ be a flexible CRR scheme, and $h$ as in Theorem 2. For simplicity assume that $N=1$. We show here how a slight modification of Korn and Müller's [13] optimization procedure allows us to reach accelerations to an order of $\mathcal{O}\left(n^{-1.5}\right)$. For this purpose only, we assume not only that the risk free rate $r$, the volatility $\sigma$, the maturity $T$ and integer $p$ are constant, but also that the payoff $h$ and the current value of the underlying asset $x$ are fixed. In other words, only $\lambda$ and $\mathfrak{f}^{(n)}$ are seen as variables. A glance at (1.4) reveals that $\operatorname{Err}_{T}^{n}(h)(x)$ can be written as

$$
\operatorname{Err}_{T}^{n}(h)(x)=\frac{P(\lambda)+Q\left(\mathfrak{f}^{(n)}\right)}{n}+\mathcal{O}\left(n^{-1.5}\right),
$$

where $\mathfrak{f}^{(n)}:=\mathfrak{f}^{(n)}\left(\lambda, \mu_{n}\right)$ is given by (1.3), and where for some constants $a, b, c, d$,

$$
\begin{gathered}
P(\lambda)=a \lambda^{2}+b \lambda+c \\
Q\left(\mathfrak{f}^{(n)}\right)=d \mathfrak{f}^{(n)}\left(1+\mathfrak{f}^{(n)}\right) .
\end{gathered}
$$

The following is admittedly a slight extension of Korn and Müller's [13] optimization procedure which shows how one can obtain an optimal scheme for our general payoff functions:

(1) Choose a constant $\chi_{0}=Q\left(\mu_{0}\right)$ for some $-1<\mu_{0}<1$,

(2) Choose $\lambda_{0}$ such that

$$
\mathfrak{m}_{\chi_{0}}:=\inf _{\lambda}\left|P(\lambda)+\chi_{0}\right|=\left|P\left(\lambda_{0}\right)+\chi_{0}\right|
$$

(3) Set $\mu_{n}:=\mu_{0}-\mathfrak{f}^{(n)}\left(\lambda_{0}, 0\right)$, and note that

$$
\mathfrak{f}^{(n)}\left(\lambda_{0}, \mu_{n}\right)=\operatorname{frac}\left(\mathfrak{f}^{(n)}\left(\lambda_{0}, 0\right)+\mu_{n}\right)=\mu_{0} .
$$


Under the binomial scheme with parameters $\left(\lambda_{0}, \mu_{n}\right)$, equation (1.6) can be rewritten as

$$
\operatorname{Err}_{T}^{n}(h)(x)=\frac{\mathfrak{m}_{\chi_{0}}}{n}+\mathcal{O}\left(n^{-\frac{3}{2}}\right) .
$$

When $\mathfrak{m}_{\chi_{0}}=0$, the convergence of the scheme has been accelerated to an order of $\mathcal{O}\left(n^{-\frac{3}{2}}\right)$, otherwise, the constant $\mathfrak{m}_{\chi_{0}}$ has been optimized.

Here the differences with [13] are that we allow $\chi_{0} \neq 0$ (which is sometimes necessary to reach $\mathfrak{m}_{\chi_{0}}=0$, as in the example provided below), and we use the error formula (1.4) to show that the remainder term is of order $O\left(n^{-\frac{3}{2}}\right)$, as opposed to $o\left(n^{-1}\right)$ in [13].

\subsection{Example and simulations.}

Example 1 (Simulation and the error formula). Consider the classical $C R R$ scheme, where $\lambda=\mu_{n}=0$, and the following payoff function

$$
h(z)= \begin{cases}z^{2} & 0 \leq z \leq \mathrm{K} \\ 1 & \mathrm{~K}<z\end{cases}
$$

which is continuous and belongs to $\mathcal{K}^{(3)}$ with $\mathrm{K}=1$. It is easy (with Maple) to calculate that

$$
\Upsilon_{T}(h, x)=\left(\frac{1}{2} \Delta_{2}-\frac{1}{3} \Delta_{3}+\frac{1}{4} \Delta_{4}\right) \mathcal{A}+\frac{1}{24} \frac{4 \Delta_{3}-5 \Delta_{4}}{\sigma \sqrt{T}} \mathcal{B}+\frac{1}{24} \frac{\Delta_{4}}{T \sigma^{2}} \mathcal{C},
$$

where

$$
\begin{aligned}
& \mathcal{A}=x^{2} e^{T\left(r-\sigma^{2}\right)}\left(c+e^{\frac{1}{2} a^{2}}\right), \\
& \mathcal{B}=x^{2} e^{T\left(r-\sigma^{2}\right)}\left(\frac{-\sqrt{2} d+a c \sqrt{\pi}+a \sqrt{\pi} e^{\frac{1}{2} a^{2}}}{\sqrt{\pi}}\right), \\
& \mathcal{C}=x^{2} e^{T\left(r-\sigma^{2}\right)}\left(\frac{-b \sqrt{2} d-a \sqrt{2} d+a^{2} \sqrt{\pi} c+e^{\frac{1}{2} a^{2}} a^{2} \sqrt{\pi}}{\sqrt{\pi}}\right),
\end{aligned}
$$

and $a=2 \sigma \sqrt{T}, b=\eta_{T}\left(\frac{\mathrm{K}}{x}\right), c=e^{\frac{1}{2} a^{2}} \operatorname{erf}\left(\frac{b-a}{\sqrt{2}}\right), d=\exp \left(-\frac{1}{2} b(-2 a+b)\right)$. In figure 1 , we set $r=0.08, \sigma=0.5, T=1$ and $x=1.1$ and, in accordance with Theorem 2, $n^{1.5}\left(\operatorname{Err}_{T}^{n} h(x)-\frac{\Upsilon_{T}(h)(x)}{n}-\Delta h^{\prime}(\mathrm{K}) \frac{\Lambda_{\mathrm{K}}^{n}(x)}{n}\right)$ is bounded.

Example 2 (Optimal Scheme). We use the same payoff function $h$ of example 1 , as well as $r=0.08, \sigma=0.5, T=1$ and $x=1.1$. Hence, everything is fixed except $\lambda$ and $\mu_{n}$. Reusing the formula of example 1, we calculate that the general first-order error formula, can be rewritten as

$$
\operatorname{Err}_{T}^{n}(h)(x)=\frac{a \lambda^{2}+b \lambda+c+d \mathfrak{f}^{(n)}\left(1+\mathfrak{f}^{(n)}\right)}{n}+\mathcal{O}\left(n^{-1.5}\right),
$$

where

$$
\begin{gathered}
a=-0.031544554932975475877, \quad b=0.015054127355591099077, \\
c=0.084196334462544764572, \quad d=-0.73282116693588932807 .
\end{gathered}
$$




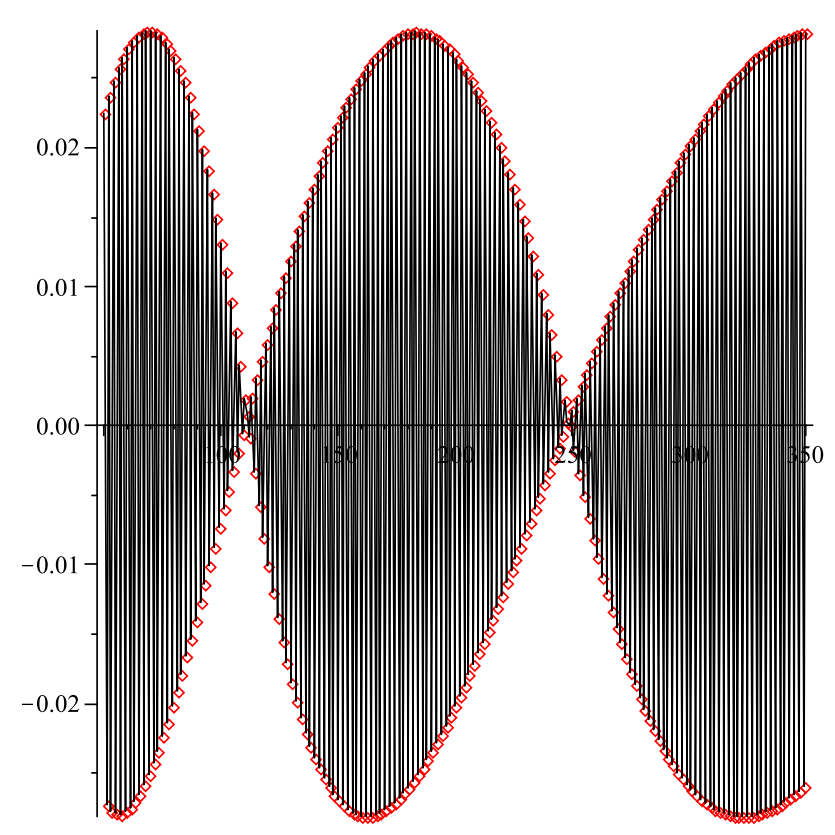

FIGURE 1. The quantity $n^{1.5}\left(\operatorname{Err}_{T}^{n} h(x)-\frac{\Upsilon_{T}(h)(x)}{n}-\right.$ $\left.\Delta h^{\prime}\left(\mathrm{K}_{1}\right) \frac{\Lambda_{\mathrm{K}_{1}}^{n}(x)}{n}\right)$ oscillates rapidly but remains bounded.

Choose $\chi_{0}=-\frac{d}{4}$, and note that $\chi_{0}=Q\left(\mu_{0}\right)$ with $\mu_{0}=-0.5$. Note also that in equation (1.7), $\mathfrak{m}_{\chi_{0}}=0$ is attained with $\lambda_{0}=1.8896959961364908175$. Letting $\mu_{n}=\mu_{0}-\mathfrak{f}^{(n)}\left(\lambda_{0}, 0\right)$, the flexible scheme $\xi^{(n)}$ with parameters $\lambda_{0}$ and $\mu_{n}$ satisfies $\operatorname{Err}_{T}^{n}(h)(x)=\mathcal{O}\left(n^{-1.5}\right)$. The convergence is illustrated in figure 2.

1.4. Settings and notation. The following lists some assumptions and notation used throughout the rest of this paper.

Constants $r, \sigma, T, p$ and $\mathcal{L}$ : We study the convergence of options with payoffs $h$ in $\mathcal{K}_{p}^{(3)}$, where $p \geq 1$ is some integer, when the geometric Brownian motion is approximated by flexible binomial schemes $\left\{\xi^{(n)}\right\}$, which depend on parameters $\lambda$ and $\mu_{n}$. We suppose that $\left|\mu_{n}\right| \leq \mathcal{L}$, for some $\mathcal{L}$. Parameters $\lambda, r, \sigma, T, p$ and $\mathcal{L}$ are fixed throughout this paper, and expressions in terms of these parameters are considered constants.

Independence of $\xi$ and $\xi^{(n)}$ : We assume that $\xi$ and $\xi^{(n)}$ are independent.

Time steps $t_{m}$ : Given $n, t_{m}$ denotes the $m^{\text {th }}$ time step, or in other words, $t_{m}=m \frac{T}{n}$.

Discounted expectations $\mathcal{E}$ and $\mathcal{E}^{n}$ : for every $t, x \geq 0$ and polynomially bounded function $h$, we denote $\mathcal{E}_{t} h(x):=e^{-r t} E_{x}\left(h\left(\xi_{t}\right)\right)$, and similarly $\mathcal{E}_{t}^{n} h(x):=e^{-r t} E_{x}\left(h\left(\xi_{t}^{(n)}\right)\right)$. Note that $\mathcal{E}$ and $\mathcal{E}^{n}$ simply 


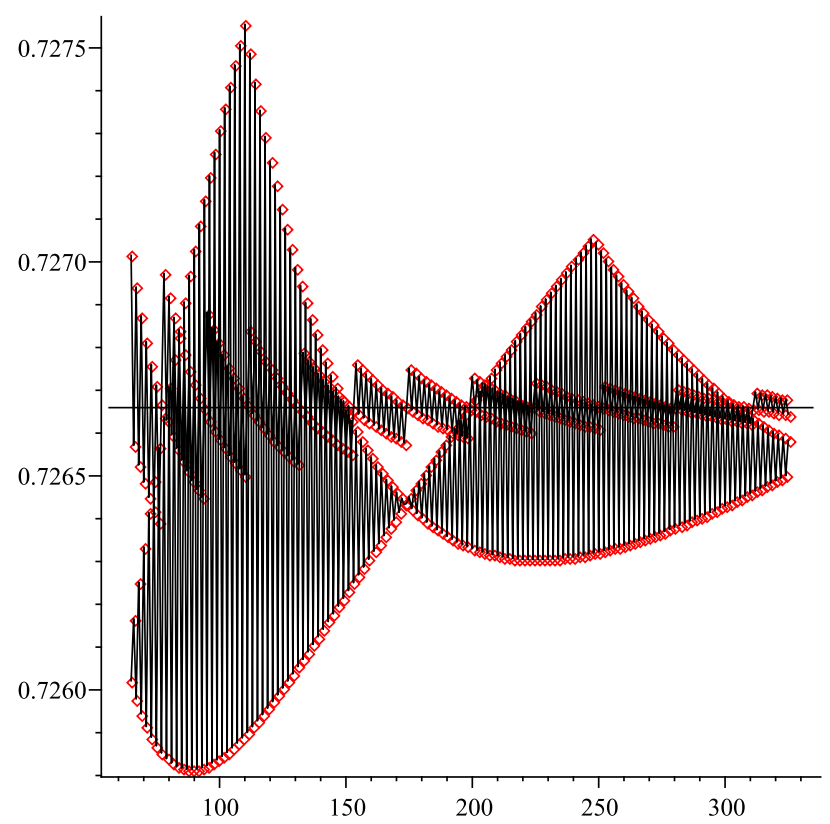

Figure 2. The value of the option as a function of $n$ for both the optimal scheme and the classical CRR scheme of example 2. The horizontal line is the value of the option in the Black-Scholes model.

denote the discounted expectation. They are semigroup operators: $\mathcal{E}_{t+s} h=\mathcal{E}_{t} \mathcal{E}_{s} h$ and $\mathcal{E}_{t+s}^{n} h=\mathcal{E}_{t}^{n} \mathcal{E}_{s}^{n} h$. Because $\xi$ and $\xi^{(n)}$ are independent, $\mathcal{E}$ and $\mathcal{E}^{n}$ commute: $\mathcal{E}_{t}^{n} \mathcal{E}_{s} h=\mathcal{E}_{s} \mathcal{E}_{t}^{n} h$.

The error $\operatorname{Err}^{n}$ : We denote $\operatorname{Err}_{t}^{n}(h)(x):=\mathcal{E}_{t} h(x)-\mathcal{E}_{t}^{n} h(x)$. Whenever possible, we write $\operatorname{Err}_{t}^{n} f(x)$ instead of $\operatorname{Err}_{t}^{n}(f)(x)$. Note that the operator $\operatorname{Err}^{n}$ commutes with $\mathcal{E}$ and $\mathcal{E}^{n}$ and therefore with itself.

The identity function $I$ and the symbols $\delta_{k}^{(n)}$ and $\Delta_{k}^{(n)}$ : We denote by $I$ the identity operator: $I(z):=z$, for every $z$. Among other things this allows us to define expressions such as

$\operatorname{Err}_{\frac{T}{n}}^{n}\left(\int_{1}^{I} g(u) d u\right)(x)=e^{-r \frac{T}{n}} E_{x}\left(\int_{1}^{\xi_{\frac{T}{n}}} g(u) d u-\int_{1}^{\xi_{\frac{T}{n}}^{(n)}} g(u) d u\right)$,

and for any integer $k \geq 0$,

$$
\begin{aligned}
\delta_{k}^{(n)} & :=\mathcal{E}_{\frac{T}{n}}^{n}\left(|I-1|^{k}\right)(1)=e^{-r \frac{T}{n}} E_{1}\left(\left|\xi_{\frac{T}{n}}^{(n)}-1\right|^{k}\right), \\
\Delta_{k}^{(n)} & :=\operatorname{Err}_{\frac{T}{n}}^{n}\left((I-1)^{k}\right)(1)=e^{-r \frac{T}{n}} E_{1}\left(\left(\xi_{\frac{T}{n}}-1\right)^{k}-\left(\xi_{\frac{T}{n}}^{(n)}-1\right)^{k}\right) .
\end{aligned}
$$

Note that $\Delta_{1}^{(n)}=0$ because both $\xi$ and $\xi^{(n)}$ are risk neutral. 
A function $\chi_{p}$ on $K_{p}^{(3)}$ : Given $h$ in $\mathcal{K}_{p}^{(3)}$, and a partition $0<K_{1}<$ $\ldots<\mathrm{K}_{N}<\infty$ of $[0, \infty)$, for which $h$ is $C^{(3)}$ when restricted to the closed intervals defined by this partition, $\chi_{p}$ is defined by:

$$
\chi_{p}(h):=\|h\|_{p}^{(3)}+\sum_{\ell=1}^{N} \sum_{j=0}^{2} \sum_{k=0}^{2}\left(\mathrm{~K}_{\ell}\right)^{j}\left|\Delta h^{(k)}\left(\mathrm{K}_{\ell}\right)\right| .
$$

1.5. Proof of Theorem 2. Using (1.2) to split the payoff function $h$ into a sum of call options and a continuously differentiable function $g$ in $\mathcal{K}_{p}^{(3)}$, one gets

$$
\operatorname{Err}_{T}^{n}(h)(x)=\sum_{\ell=1}^{N} \Delta h^{\prime}\left(\mathrm{K}_{\ell}\right) \Lambda_{T}^{n}\left(\mathrm{~K}_{\ell}, x\right)+\operatorname{Err}_{T}^{n}(g)(x),
$$

where $\Lambda_{T}^{n}(\mathrm{~K}, x)=\operatorname{Err}_{T}^{n}(\max (I-\mathrm{K}, 0))(x)$ is the error for a call option with strike $K$. Because smooth functions are undoubtedly easier to deal with, we replace $g$ by $\mathcal{E}_{\frac{T}{n}} g$, which is the option itself evaluated over one single time step. This provides a new smoothed payoff which is infinitely differentiable. This smoothing of $g$ splits the error, $\operatorname{Err}_{T}^{n} g$, into a sum of two terms: $\operatorname{Err}_{T}^{n}\left(g-\mathcal{E}_{\frac{T}{n}} g\right)$, the error coming from the payoff smoothing procedure itself, and $\operatorname{Err}_{T}^{n} \mathcal{E}_{\frac{T}{n}}$ g, the error of the smoothed payoff. The fact that the payoff smoothing error is negligible, that is of order $\mathcal{O}\left(n^{-\frac{3}{2}}\right)$, is what Theorem 6 says. As for the smoothed payoff error, Theorem 4 says that

$$
\operatorname{Err}_{T}^{n}\left(\mathcal{E}_{\frac{T}{n}} g\right)(x)=\frac{1}{n} \sum_{k=2}^{4} \frac{\Delta_{k}}{k !} x^{k} \frac{\partial^{k}}{\partial x^{k}} \mathcal{E}_{T} g(x)+\mathcal{O}\left(n^{-\frac{3}{2}}\right) .
$$

Using the representation formulae for the derivatives $\frac{\partial^{k}}{\partial x^{k}} \mathcal{E}_{T} g(x)$ given in Theorem 5, tedious arithmetic simplifications allow to rewrite the above as

$$
\operatorname{Err}_{T}^{n}\left(\mathcal{E}_{\frac{T}{n}} g\right)(x)=\Upsilon_{T}(g)(x)+\mathcal{O}\left(n^{-\frac{3}{2}}\right) .
$$

Since $g^{\prime \prime}=h^{\prime \prime}$ then $\Upsilon_{T}(g)(x)=\Upsilon_{T}(h)(x)$, which completes the proof.

1.6. Outline of the paper. Theorem 2 establishes a formula for the error $\operatorname{Err}_{T}^{n}(h)(x)$, when the continuous payoff function $h$ belongs to $\mathcal{K}_{p}^{(3)}$. In the proof of Theorem 2, after $h$ has been split into a sum of call options and a function $g$ in $C^{(1)} \cap \mathcal{K}_{p}^{(3)}$, finding the formula for $\operatorname{Err}_{T}^{n}(h)(x)$ requires to show that $\operatorname{Err}_{T}^{n}\left(g-\mathcal{E}_{\frac{T}{n}} g\right)(x)$ is negligible, and to find an error formula for $\operatorname{Err}_{T}^{n}\left(\mathcal{E}_{\frac{T}{n}} g\right)(x)$, more specifically to show (1.9). The latter task is the subject of Theorem 4 . We now sketch how various parts of this paper fit together to establish (1.9). Let us point out that Theorem 4 comes naturally - and in great generality - from a localization formula and an expansion formula of these local errors, used in conjunction with our representation formulae. A local error refers here to an error when the maturity is $\frac{T}{n}$, 
and error localization refers to expressing an error as a sum of (discounted expected) local errors and a sum of errors of local errors.

Because of the localization formula (Theorem 3), we have

$$
\operatorname{Err}_{T}^{n} \mathcal{E}_{\frac{T}{n}} g=\sum_{j=0}^{n-1} \mathcal{E}_{T-t_{j+1}}\left(\operatorname{Err}_{\frac{T}{n}}^{n} \mathcal{E}_{t_{j}} \mathcal{E}_{\frac{T}{n}} g\right)-\sum_{j=0}^{n-1} \operatorname{Err}_{T-t_{j+1}}^{n}\left(\operatorname{Err}_{\frac{T}{n}}^{n} \mathcal{E}_{t_{j}} \mathcal{E}_{\frac{T}{n}} g\right) .
$$

Thus, because $\operatorname{Err}^{n}$ and $\mathcal{E}$ commute, and because $\mathcal{E}$ is a semigroup,

$$
\operatorname{Err}_{T}^{n} \mathcal{E}_{T} g=\sum_{j=1}^{n} \operatorname{Err}_{\frac{T}{n}}^{n} \mathcal{E}_{T} g-\sum_{j=1}^{n} \operatorname{Err}_{T-t_{j}}^{n}\left(\operatorname{Err}_{\frac{T}{n}}^{n} \mathcal{E}_{t_{j}} g\right)
$$

To avoid technicalities, let us temporarily ignore the facts that local errors $\operatorname{Err}_{\frac{T}{n}}^{n} \mathcal{E}_{t_{j}} g(x)$ depend on the payoff $\mathcal{E}_{t_{j}} g$ and on the initial value $x$ of the underlying asset, and that the $\mathcal{O}$ terms are not uniform in the payoff and the initial value of the underlying asset. Then, as pointed out in Remark 3, we can rewrite our local error expansion formula for $\operatorname{Err}_{\frac{T}{n}}^{n} \mathcal{E}_{t_{j}} g$ as

$$
\operatorname{Err}_{\frac{T}{n}}^{n} \mathcal{E}_{t_{j}} g=\frac{1}{n^{2}} \sum_{k=2}^{4} \frac{\Delta_{k}}{k !} x^{k} \frac{\partial^{k}}{\partial x^{k}} \mathcal{E}_{t_{j}} g+\mathcal{O}\left(n^{-\frac{5}{2}}\right),
$$

from which we obtain the following error localization expansion formula for $\operatorname{Err}_{T}^{n} \mathcal{E}_{\frac{T}{n}} g$ :

$$
\begin{aligned}
\operatorname{Err}_{T}^{n} \mathcal{E}_{\frac{T}{n}} g & =\frac{1}{n} \sum_{k=2}^{4} \frac{\Delta_{k}}{k !} x^{k} \frac{\partial^{k}}{\partial x^{k}} \mathcal{E}_{T} g \\
& -\sum_{k=2}^{4} \sum_{j=1}^{n} \frac{\Delta_{k}}{k ! n^{2}} \operatorname{Err}_{T-t_{j}}^{n}\left(I^{k} \frac{\partial^{k}}{\partial x^{k}} \mathcal{E}_{t_{j}} g\right)+\mathcal{O}\left(n^{-\frac{3}{2}}\right) .
\end{aligned}
$$

But Lemma 3 shows that

$$
\operatorname{Err}_{T-t_{j}}^{n}\left(I^{k} \frac{\partial^{k}}{\partial x^{k}} \mathcal{E}_{t_{j}} g\right)={\sqrt{t_{j}}}^{-(k-1)} \mathcal{O}\left(n^{-1}\right),
$$

and therefore simple calculations give, as wanted,

$$
\operatorname{Err}_{T}^{n} \mathcal{E}_{\frac{T}{n}} g=\frac{1}{n} \sum_{k=2}^{4} \frac{\Delta_{k}}{k !} x^{k} \frac{\partial^{k}}{\partial x^{k}} \mathcal{E}_{T} g+\mathcal{O}\left(n^{-\frac{3}{2}}\right) .
$$

To complete the outline of this paper, let us mention that section 2 gathers the results about localization, section 3 deals with the smoothed payoff error, while section 4 establishes our representation formulae for the derivatives of European options. The fact that payoff smoothing errors are negligible is proved in section 5 . The appendix contains auxiliary results, including a list of some simple properties, P1-P5, enjoyed by all flexible CRR schemes.

Remark 2 (On the $\mathcal{O}$ notation). In the rest of the paper, unless otherwise mentioned, the $\mathcal{O}$ notation is uniform. By this we mean that if $A, B$ and $C \geq 0$ are real-valued, then the expression $A=B+C \mathcal{O}\left(n^{-1}\right)$ means that 
there exists a constant $Q$, which may depend only on our parameters $r, \sigma$, $T, p$ and $\mathcal{L}$, such that $|A-B| \leq C Q n^{-1}$.

\section{LOCAL ERRORS AND ERROR LOCALIZATION}

The localization of errors and the treatment of local errors plays an important role in this paper. The idea stems from a step-by-step approach corresponding to the steps in the binomial tree approach. From a binary tree point of view, the equation

$$
\mathcal{E}_{\frac{T}{n}}^{n} h(x)=h(x u(n)) p(n)+h(x d(n))(1-p(n))
$$

corresponds to calculating the discounted expectation in a single time-step tree, while $\mathcal{E}_{t_{j}}^{n} h$ does this with a $j$ time-step tree. Programming this binary tree approach naturally leads to recursive calls to the operator $\mathcal{E}_{\frac{T}{n}}^{n}$ :

$$
\mathcal{E}_{t_{j+1}}^{n} h=\mathcal{E}_{\frac{T}{n}}^{n}\left(\mathcal{E}_{t_{j}}^{n} h\right)=\mathcal{E}_{\frac{T}{n}}^{n}\left(\mathcal{E}_{\frac{T}{n}}^{n}\left(\mathcal{E}_{t_{j-1}}^{n} h\right)\right),
$$

and more generally

$$
\mathcal{E}_{t_{j+1}}^{n} h=\overbrace{\mathcal{E}_{\frac{T}{n}}^{n}\left(\mathcal{E}_{\frac{T}{n}}^{n}\left(\ldots\left(\mathcal{E}_{\frac{T}{n}}^{n} h\right)\right)\right)}^{j+1 \text { times }} .
$$

This equation reveals the semigroup and commutation properties of $\mathcal{E}^{n}$,

$$
\mathcal{E}_{t_{j}+t_{k}}^{n} h=\mathcal{E}_{t_{j}}^{n} \mathcal{E}_{t_{k}}^{n} h=\mathcal{E}_{t_{k}}^{n} \mathcal{E}_{t_{j}}^{n} h,
$$

which extend to

$$
\mathcal{E}_{t_{j}}^{n} \mathcal{E}_{t_{k}} h=\mathcal{E}_{t_{k}} \mathcal{E}_{t_{j}}^{n} h
$$

This semigroup property is equivalent to the fact that one can value an option in two steps: if the expiry date of the option is $T$, and $t_{j}$ is some intermediate time, one can first value the option with a maturity of $T-t_{j}$, which depends one the stock price, and then considering that as an option with maturity $t_{j}$, find its value at time 0 . More generally, one can first evaluate the option with a maturity of $\frac{T}{n}$, use this as the payoff of another option with maturity $\frac{T}{n}$, and continue this way until the full maturity $T$ has been reached, just as in (2.1). In this binary tree recursive implementation, one starts by calculating $\mathcal{E}_{\frac{T}{n}}^{n} h$. If the ultimate goal is to estimate $\mathcal{E}_{T} h$ through $\mathcal{E}_{T}^{(n)} h$, it is natural to keep track of the error resulting from this very first step:

$$
\mathcal{E}_{\frac{T}{n}}^{n} h=\mathcal{E}_{\frac{T}{n}} h-\left(\mathcal{E}_{\frac{T}{n}} h-\mathcal{E}_{\frac{T}{n}}^{n} h\right)=\mathcal{E}_{\frac{T}{n}} h-\operatorname{Err}_{\frac{T}{n}}^{n} h .
$$

Then comes the second call to $\mathcal{E}_{\frac{T}{n}}^{n}$ :

$$
\mathcal{E}_{2 \frac{T}{n}}^{n} h=\mathcal{E}_{\frac{T}{n}}^{n}\left(\mathcal{E}_{\frac{T}{n}}^{n} h\right)=\mathcal{E}_{\frac{T}{n}}^{n}\left(\mathcal{E}_{\frac{T}{n}} h-\operatorname{Err}_{\frac{T}{n}}^{n} h\right)=\mathcal{E}_{\frac{T}{n}}^{n} \mathcal{E}_{\frac{T}{n}} h-\mathcal{E}_{\frac{T}{n}}^{n}\left(\operatorname{Err}_{\frac{T}{n}}^{n} h\right) .
$$


Note that

$$
\mathcal{E}_{\frac{T}{n}}^{n} \mathcal{E}_{\frac{T}{n}} h=\mathcal{E}_{\frac{T}{n}} \mathcal{E}_{\frac{T}{n}} h-\left(\mathcal{E}_{\frac{T}{n}} \mathcal{E}_{\frac{T}{n}} h-\mathcal{E}_{\frac{T}{n}}^{n} \mathcal{E}_{\frac{T}{n}} h\right)=\mathcal{E}_{2 \frac{T}{n}} h-\operatorname{Err}_{\frac{T}{n}}^{n} \mathcal{E}_{\frac{T}{n}} h
$$

Hence,

$$
\mathcal{E}_{2 \frac{T}{n}}^{n} h=\mathcal{E}_{2 \frac{T}{n}} h-\mathcal{E}_{\frac{T}{n}}^{n}\left(\operatorname{Err}_{\frac{T}{n}}^{n} h\right)-\operatorname{Err}_{\frac{T}{n}}^{n} \mathcal{E}_{\frac{T}{n}} h .
$$

This procedure can be continued, resulting in

$$
\mathcal{E}_{t_{m}}^{n} h=\mathcal{E}_{t_{m}} h-\sum_{j=0}^{m-1} \mathcal{E}_{t_{m}-t_{j+1}}^{n}\left(\operatorname{Err}_{\frac{T}{n}}^{n} \mathcal{E}_{t_{j}} h\right) .
$$

It shows that, if the local errors can be calculated with sufficient accuracy, then the global error can be accurately estimated from these local errors. Equation (2.3) is equivalent to

$$
\operatorname{Err}_{t_{m}}^{n} h=\sum_{j=0}^{m-1} \mathcal{E}_{t_{m}-t_{j+1}}^{n}\left(\operatorname{Err}_{\frac{T}{n}}^{n} \mathcal{E}_{t_{j}} h\right) .
$$

It says that, when evaluating a European option with maturity $t_{m}$, the error with respect to the Black-Scholes model can be decomposed into the sum of the discounted expected values, with respect to $\mathcal{E}_{t_{m}-t_{j+1}}^{n}$, of the single step errors $\operatorname{Err}_{\frac{T}{n}}^{n} \mathcal{E}_{t_{j}} h$. Replacing the terms $\mathcal{E}_{t_{m}-t_{j+1}}^{n}$ by $\mathcal{E}_{t_{m}-t_{j+1}}$ in the righthand side of equation (2.4) results in additional error terms: the compounded errors

$$
-\operatorname{Err}_{t_{m}-t_{j+1}}^{n}\left(\operatorname{Err}_{\frac{T}{n}}^{n} \mathcal{E}_{t_{j}} h\right),
$$

which follow from the trivial identity

$$
\mathcal{E}_{t_{m}-t_{j+1}}^{n}=\mathcal{E}_{t_{m}-t_{j+1}}-\operatorname{Err}_{t_{m}-t_{j+1}}^{n} .
$$

This discussion should provide a solid intuitive background for the following Error localization formula.

Theorem 3 (Error localization formula). Let $n, m \geq 1$ be integers, and let $h$ be a polynomially bounded function. Then,

$$
\operatorname{Err}_{t_{m}}^{n} h=\sum_{j=0}^{m-1} \mathcal{E}_{t_{m}-t_{j+1}}\left(\operatorname{Err}_{\frac{T}{n}}^{n} \mathcal{E}_{t_{j}} h\right)-\sum_{j=0}^{m-1} \operatorname{Err}_{t_{m}-t_{j+1}}^{n}\left(\operatorname{Err}_{\frac{T}{n}}^{n} \mathcal{E}_{t_{j}} h\right)
$$

Proof. First we prove (2.3) by induction. When $m=1,(2.3)$ coincides with (2.2), which is trivially true. Assuming that (2.3) has been established for $m=k$, we get

$$
\begin{aligned}
\mathcal{E}_{t_{k+1}}^{n} h & =\mathcal{E}_{t_{1}}^{n} \mathcal{E}_{t_{k}} h-\sum_{j=0}^{k-1} \mathcal{E}_{t_{1}}^{n} \mathcal{E}_{t_{k}-t_{j+1}}^{n}\left(\operatorname{Err}_{\frac{T}{n}}^{n} \mathcal{E}_{t_{j}} h\right) \\
& =\mathcal{E}_{t_{1}}^{n} \mathcal{E}_{t_{k}} h-\sum_{j=0}^{k-1} \mathcal{E}_{t_{k+1}-t_{j+1}}^{n}\left(\operatorname{Err}_{\frac{T}{n}}^{n} \mathcal{E}_{t_{j}} h\right) .
\end{aligned}
$$


Using (2.2) with $h$ replaced by $\mathcal{E}_{t_{k}} h$, we obtain

$$
\mathcal{E}_{t_{k+1}}^{n} h=\mathcal{E}_{t_{k+1}} h-\operatorname{Err}_{\frac{T}{n}}^{n} \mathcal{E}_{t_{k}} h-\sum_{j=0}^{k-1} \mathcal{E}_{t_{k+1}-t_{j+1}}^{n}\left(\operatorname{Err}_{\frac{T}{n}}^{n} \mathcal{E}_{t_{j}} h\right) .
$$

This rewrites as (2.3), yielding (2.4). Using

$$
\mathcal{E}_{t_{m}-t_{j+1}}^{n}\left(\operatorname{Err}_{\frac{T}{n}}^{n} \mathcal{E}_{t_{j}} h\right)=\mathcal{E}_{t_{m}-t_{j+1}}\left(\operatorname{Err}_{\frac{T}{n}}^{n} \mathcal{E}_{t_{j}} h\right)-\operatorname{Err}_{t_{m}-t_{j+1}}^{n}\left(\operatorname{Err}_{\frac{T}{n}}^{n} \mathcal{E}_{t_{j}} h\right),
$$

equation (2.5) follows from (2.4) in the most trivial manner.

Local errors can be analyzed in a great variety of ways, including a simple Taylor expansion as in Lemma 1 below, where the expression $\sum_{k=2}^{N}$ is understood to vanish in the case $N<2$.

Lemma 1 (Local error expansion formula). For every integer $N \geq 0, p \geq 1$, $g \in C^{(N)} \cap \mathcal{K}_{p}^{(N+1)}$ and $x \geq 0$,

$$
\operatorname{Err}_{\frac{T}{n}}^{n} g(x)=\sum_{k=2}^{N} \frac{\Delta_{k}^{(n)} x^{k} g^{(k)}(x)}{k !}+\mathcal{R}_{\frac{T}{n}}^{n, N}\left(I^{N+1} g^{(N+1)}\right)(x),
$$

where for every function $\psi$,

$$
\mathcal{R}_{\frac{T}{n}}^{n, N}(\psi)(x):=\frac{1}{N !} \operatorname{Err}_{\frac{T}{n}}^{n}\left(\int_{1}^{I} \frac{\psi(x u)(I-u)^{N}}{u^{N+1}} d u\right)(1) .
$$

Proof. Recall that the Taylor expansion of $g(y)$ around $x$ is

$$
g(y)-g(x)=\sum_{k=1}^{N} \frac{g^{(k)}(x)}{k !}(y-x)^{k}+\frac{1}{N !} \int_{x}^{y} g^{(N+1)}(u)(y-u)^{N} d u .
$$

Using the (discounted expected) Taylor expansions of $g\left(x \xi_{\frac{T}{n}}\right)$ and $g\left(x \xi_{\frac{T}{n}}^{(n)}\right)$ around $x$ in

$$
\operatorname{Err}_{\frac{T}{n}}^{n} g(x)=e^{-r \frac{T}{n}} E_{1}\left(\left(g\left(x \xi_{\frac{T}{n}}\right)-g(x)\right)-\left(g\left(x \xi_{\frac{T}{n}}^{(n)}\right)-g(x)\right)\right),
$$

one gets (2.6) after a simple manipulation of the remainder.

Remark 3 (Order of the remainder $\mathcal{R}_{\frac{T}{n}}^{n, N}$ ). If for some constants $\alpha \geq 0$ and $\beta \geq 1$

$$
\left|x^{N+1} g^{(N+1)}(x)\right| \leq \alpha\left(1+x^{\beta}\right)
$$

then

$$
\begin{aligned}
\left|\mathcal{R}_{\frac{T}{n}}^{n, N}\left(I^{N+1} g^{(N+1)}\right)(x)\right| & \leq \frac{\alpha}{N !} \operatorname{Err}_{\frac{T}{n}}^{n}\left(\left|\int_{1}^{I} u^{-(N+1)}(I-u)^{N} d u\right|\right)(1) \\
& +\frac{\alpha x^{\beta}}{N !} \operatorname{Err}_{\frac{T}{n}}^{n}\left(\left|\int_{1}^{I} u^{\beta-(N+1)}(I-u)^{N} d u\right|\right)(1)
\end{aligned}
$$


and, because of property P5 in section 6,

$$
\left|\mathcal{R}_{\frac{T}{n}}^{n, N}\left(I^{N+1} g^{(N+1)}\right)(x)\right|=\alpha\left(1+x^{\beta}\right) \mathcal{O}\left(n^{-\frac{N+1}{2}}\right) .
$$

Now a glance at the error localization formula reveals that we deal with local errors, $\operatorname{Err}_{\frac{T}{n}}^{n} \mathcal{E}_{s} g$, where the payoff has the form $\mathcal{E}_{s} g$ for some time step s. As pointed ${ }^{\frac{1}{n}}$ out in section 1.6, we are interested in the case $s>0$, and $g$ in $C^{(1)} \cap \mathcal{K}_{p}^{(3)}$. But in this case, the European option derivative representation formulae (see Remark 6 below) guarantees that

$$
\left|I^{5} \frac{\partial^{5}}{\partial x^{5}} \mathcal{E}_{s} g(x)\right| \leq\|g\|_{p}^{(2)}(\sqrt{s})^{-3}\left(1+x^{p+2}\right)
$$

and thus

$$
\left|\mathcal{R}_{\frac{T}{n}}^{n, 4}\left(I^{5} \frac{\partial^{5}}{\partial x^{5}} \mathcal{E}_{s} g\right)(x)\right|=\|g\|_{p}^{(2)}(\sqrt{s})^{-3}\left(1+x^{p+2}\right) \mathcal{O}\left(n^{-\frac{5}{2}}\right) .
$$

Because of Theorem 3, the error $\operatorname{Err}_{t_{m}}^{n} g(x)$ can be decomposed into two components which need to be analyzed separately: (1) the main term of the error, denoted $\operatorname{MErr}_{t_{m}}^{n} g(x)$, which is the sum, over $j=0, \ldots, m-1$, of the local errors $\mathcal{E}_{t_{m}-t_{j+1}}\left(\operatorname{Err}_{\frac{T}{n}}^{n} \mathcal{E}_{t_{j}} g\right)$; (2) the compounded error term, denoted $\operatorname{CErr}_{t_{m}}^{n} g(x)$, which is the sum, over $j=0, \ldots, m-1$, of these errors of local errors $\operatorname{Err}_{t_{m}-t_{j+1}}^{n}\left(\operatorname{Err}_{\frac{T}{n}}^{n} \mathcal{E}_{t_{j}} g\right)$. In other words,

$$
\begin{aligned}
& \operatorname{MErr}_{t_{m}}^{n} g(x):=\sum_{j=0}^{m-1} \mathcal{E}_{t_{m}-t_{j+1}}\left(\operatorname{Err}_{\frac{T}{n}}^{n} \mathcal{E}_{t_{j}} g\right)(x), \\
& \operatorname{CErr}_{t_{m}}^{n} g(x):=\sum_{j=0}^{m-1} \operatorname{Err}_{t_{m}-t_{j+1}}^{n}\left(\operatorname{Err}_{\frac{T}{n}}^{n} \mathcal{E}_{t_{j}} g\right)(x) .
\end{aligned}
$$

Of course we want to combine the error localization formula with the local error expansion formula obtaining:

Proposition 1 (Error localization expansion formula). Let $M \geq 0$ be an integer, let $0<t_{m} \leq T$ be the $m^{\text {th }}$ time step, and assume that $g$ belongs to $C^{(M)} \cap \mathcal{K}_{p}^{(M+1)}$. Then, for every integer $N \geq 0$ and for every $x>0$,

$$
\operatorname{Err}_{t_{m}}^{n} g(x)=\operatorname{MErr}_{t_{m}}^{n} g(x)-\operatorname{CErr}_{t_{m}}^{n} g(x)
$$


where

$$
\begin{aligned}
\operatorname{MErr}_{t_{m}}^{n} g(x) & =m \sum_{k=2}^{N} \frac{\Delta_{k}^{(n)}}{k !} x^{k} \frac{\partial^{k}}{\partial x^{k}} \mathcal{E}_{t_{m-1}}(g)(x) \\
& +m \mathcal{R}_{\frac{T}{n}}^{n, N}\left(I^{N+1} \frac{\partial^{N+1}}{\partial x^{N+1}} \mathcal{E}_{t_{m-1}} g\right)(x) \\
\mathrm{CErr}_{t_{m}}^{n} g(x)= & \sum_{j=1}^{m-1} \sum_{k=2}^{N} \frac{\Delta_{k}^{(n)}}{k !} \operatorname{Err}_{t_{m}-t_{j+1}}^{n}\left(I^{k} \frac{\partial^{k}}{\partial x^{k}} \mathcal{E}_{t_{j}} g\right)(x) \\
& +\sum_{j=1}^{m-1} \mathcal{R}_{\frac{T}{n}}^{n, N}\left(\operatorname{Err}_{t_{m}-t_{j+1}}^{n}\left(I^{N+1} \frac{\partial^{N+1}}{\partial x^{N+1}} \mathcal{E}_{t_{j}} g\right)\right)(x) \\
& +\sum_{k=2}^{M} \frac{\Delta_{k}^{(n)}}{k !} \operatorname{Err}_{t_{m-1}}^{n}\left(I^{k} \frac{\partial^{k}}{\partial x^{k}} g\right)(x) \\
& +\mathcal{R}_{\frac{T}{n}}^{n, M}\left(\operatorname{Err}_{t_{m-1}}^{n}\left(I^{M+1} \frac{\partial^{M+1}}{\partial x^{M+1}} g\right)\right)(x) .
\end{aligned}
$$

Proof. Note that, for every $s>0, \mathcal{E}_{s} g$ belongs to $C^{(N)} \cap \mathcal{K}_{p}^{(N+1)}$, for every integer $N \geq 0$. The result is obtained by a simple combination of the error localization formula and the local error expansion formula, using the facts that for every pair of steps $t_{\ell}$ and $t_{m}$, every polynomially bounded function $\psi$ and every integer $k \geq 0$, the following hold:

(1) $\mathcal{E}_{t_{\ell}}$ and $\operatorname{Err}_{\frac{T}{n}}^{n}$ commute,

$$
\mathcal{E}_{t_{\ell}} \operatorname{Err}_{\frac{T}{n}}^{n} \psi=\operatorname{Err}_{\frac{T}{n}}^{n} \mathcal{E}_{t_{\ell}} \psi
$$

(2) by independence and Fubini's theorem, $\operatorname{Err}_{t_{\ell}}^{n}$ and $\mathcal{R}_{\frac{T}{n}}^{n, N}$ also commute,

$$
\operatorname{Err}_{t_{\ell}}^{n}\left(\mathcal{R}_{\frac{T}{n}}^{n, N}(\psi)\right)=\mathcal{R}_{\frac{T}{n}}^{n, N}\left(\operatorname{Err}_{t_{\ell}}^{n}(\psi)\right)
$$

(3) because of Lemma 3 below,

$$
\mathcal{E}_{t_{\ell}}\left(I^{k} \frac{\partial^{k}}{\partial x^{k}} \mathcal{E}_{t_{m}} \psi\right)=I^{k} \frac{\partial^{k}}{\partial x^{k}} \mathcal{E}_{t_{\ell}} \mathcal{E}_{t_{m}} \psi=I^{k} \frac{\partial^{k}}{\partial x^{k}} \mathcal{E}_{t_{\ell}+t_{m}} \psi
$$

Remark 4. We will use the error localization expansion formula with $N=$ 4. The reason for this is, in essence, that if $g$ belongs to $C^{(4)} \cap \mathcal{K}_{p}^{(5)}$, then each of the remainders - the $\mathcal{R}$-terms - in formulae (2.7) and (2.8) are of order $n^{-\frac{5}{2}}$, which makes them collectively of order $n^{-\frac{3}{2}}$, and thus negligible. 


\section{THE SMOOTHED PAYOFF ERROR}

As noted earlier - in section 1.6 - we are particularly interested in the error $\operatorname{Err}_{T}^{n} \mathcal{E}_{\frac{T}{n}} g(x)$, which decomposes into

$$
\operatorname{Err}_{T}^{n} \mathcal{E}_{\frac{T}{n}} g(x)=\operatorname{MErr}_{T}^{n} \mathcal{E}_{\frac{T}{n}} g(x)-\mathrm{CErr}_{T}^{n} \mathcal{E}_{\frac{T}{n}} g(x) .
$$

Now thanks to the European option derivative representation formulae, Theorem 5 below, for every integer $k \geq 0$,

$$
\left|x^{k} \frac{\partial^{k}}{\partial x^{k}} \mathcal{E}_{T} g(x)\right| \leq\|g\|_{p}^{(2)}\left(1+x^{p+2}\right) \mathcal{O}(1) .
$$

Therefore, using property P3, $\Delta_{k}^{(n)}=\left(\frac{1}{n}\right)^{2} \Delta_{k}+\mathcal{O}\left(n^{-\frac{5}{2}}\right)$, and Remark 3, one rewrites the main term of the error as

$$
\operatorname{MErr}_{T}^{n} \mathcal{E}_{\frac{T}{n}} g(x)=\frac{1}{n} \sum_{k=2}^{4} \frac{\Delta_{k}}{k !} x^{k} \frac{\partial^{k}}{\partial x^{k}} \mathcal{E}_{T} g(x)+\|g\|_{p}^{(2)}\left(1+x^{p+2}\right) \mathcal{O}\left(n^{-\frac{3}{2}}\right) .
$$

As for the compounded error term, $\mathrm{CErr}_{T}^{n} \mathcal{E}_{\frac{T}{n}} g(x)$, the error localization expansion formula gives (with $N=M=4$ )

$$
\begin{aligned}
\operatorname{CErr}_{T}^{n} \mathcal{E}_{\frac{T}{n}} g(x) & =\sum_{j=1}^{n} \sum_{k=2}^{4} \frac{\Delta_{k}^{(n)}}{k !} \operatorname{Err}_{T-t_{j}}^{n}\left(I^{k} \frac{\partial^{k}}{\partial x^{k}} \mathcal{E}_{t_{j}} g\right)(x) \\
& +\sum_{j=1}^{n} \mathcal{R}_{\frac{T}{n}}^{n, 4}\left(\operatorname{Err}_{T-t_{j}}^{n}\left(I^{5} \frac{\partial^{5}}{\partial x^{5}} \mathcal{E}_{t_{j}} g\right)\right)(x) .
\end{aligned}
$$

Now Lemma 4 below says that, for $0<t_{j}<T$,

$$
\operatorname{Err}_{T-t_{j}}^{n}\left(I^{k} \frac{\partial^{k}}{\partial x^{k}} \mathcal{E}_{t_{j}} g\right)=\chi_{p}(g){\sqrt{t_{j}}}^{-(k-1)} \mathcal{O}\left(n^{-1}\right)\left(1+x^{p+3}\right)
$$

where $\varkappa_{p}$ is defined by (1.8). As for the case $t_{j}=T$, it amounts only to $\mathcal{O}\left(n^{-\frac{3}{2}}\right)\|g\|_{p}^{(2)}\left(1+x^{p+2}\right)$, in formula (3.1), due to remark 3 and property P3. Thus, replacing (3.2) in (3.1) gives

$$
\begin{aligned}
\operatorname{CErr}_{T}^{n} \mathcal{E}_{\frac{T}{n}} g(x) & =\sum_{j=1}^{n-1} \sum_{k=2}^{4} \frac{O\left(n^{-2}\right)}{k !} \chi_{p}(g){\sqrt{t_{j}}}^{-(k-1)} \mathcal{O}\left(n^{-1}\right)\left(1+x^{p+3}\right) \\
& +\sum_{j=1}^{n-1} \chi_{p}(g){\sqrt{t_{j}}}^{-4} \mathcal{O}\left(n^{-1}\right)\left(1+x^{p+3}\right) \mathcal{O}\left(n^{-\frac{5}{2}}\right) \\
& +\|g\|_{p}^{(2)}\left(1+x^{p+2}\right) \mathcal{O}\left(n^{-\frac{3}{2}}\right) \\
& =\chi_{p}(g)\left(1+x^{p+3}\right) \mathcal{O}\left(n^{-\frac{3}{2}}\right) .
\end{aligned}
$$

We have proved the following result: 
Theorem 4. For every $g \in C^{(1)} \cap \mathcal{K}_{p}^{(3)}$ and every $x>0$,

$$
\operatorname{Err}_{T}^{n} \mathcal{E}_{\frac{T}{n}} g(x)=\frac{1}{n} \sum_{k=2}^{4} \frac{\Delta_{k}}{k !} x^{k} \mathcal{E}_{T}^{(k)} g(x)+\varkappa_{p}(g)\left(1+x^{p+3}\right) \mathcal{O}\left(n^{-\frac{3}{2}}\right) .
$$

Theorem 4 is a key component of the proof of Theorem 2. As pointed out in section 1.5, our first-order error formula (1.4) is obtained from (3.3) by a trivial use of the European option derivative representation formulae below. The European option derivative representation formulae and the negligibility of the payoff smoothing error are proven below.

\section{European option DERIVATIVE REPRESENTAtion FORMUlae}

Let $\phi(z)$ be the pdf of a standard normal random variable and let, as usual, $\phi^{(j)}(z)$ be its $j^{\text {th }}$ derivative. To shorten expressions, we denote $\zeta_{s}(z):=e^{\sqrt{s} \sigma z+\left(r-\frac{1}{2} \sigma^{2}\right) s}$, with which we can write

$$
\mathcal{E}_{s} h(x)=e^{-r s} \int_{-\infty}^{\infty} h\left(x \zeta_{s}(z)\right) \phi(z) d z .
$$

We will show that the derivatives of $\mathcal{E}_{s} h(x)$ can be expressed as linear combinations of smooth functions $\mathfrak{E}_{s}^{(j)} h(x)$ of the form

$$
\mathfrak{E}_{s}^{(j)} h(x):=e^{-r s} \int_{-\infty}^{\infty} h\left(x \zeta_{s}(z)\right) \phi^{(j)}(z) d z .
$$

Not only do we need expressions for the derivatives of $\mathcal{E}_{s} h(x)$, but it turns out that we also need expressions for the derivatives of $I^{k} \frac{\partial^{k}}{\partial x^{k}} \mathcal{E}_{s} h$, for integers $k \geq 0$. This motivates the notation

$$
\begin{aligned}
& \mathcal{E}_{s}^{(k)} h(x):=\frac{\partial^{k}}{\partial x^{k}} \mathcal{E}_{s} h(x), \\
& \mathcal{E}_{s}^{\langle k\rangle} h(x):=x^{k} \mathcal{E}_{s}^{(k)} h(x),
\end{aligned}
$$

and, more generally, for any function $\psi$ in $C^{(k)}$,

$$
\psi^{\langle k\rangle}(x):=x^{k} \psi^{(k)}(x) .
$$

Now let $h$ be any continuous function in $\mathcal{K}^{(1)}$ and let $s>0$. Integration by parts gives

$$
e^{-r s} \int_{-\infty}^{\infty} \zeta_{s}(z) h^{\prime}\left(x \zeta_{s}(z)\right) \phi^{(j)}(z) d z=\frac{-1}{x \sqrt{s} \sigma} \mathfrak{E}_{s}^{(j+1)} h(x),
$$

and since

$$
\frac{\partial}{\partial x} \mathfrak{E}_{s}^{(j)} h(x)=e^{-r s} \int_{-\infty}^{\infty} \zeta_{s}(z) h^{\prime}\left(x \zeta_{s}(z)\right) \phi^{(j)}(z) d z,
$$

then

$$
\frac{\partial}{\partial x} \mathfrak{E}_{s}^{(j)} h(x)=\frac{-1}{x \sqrt{s} \sigma} \mathfrak{E}_{s}^{(j+1)} h(x)
$$


Thus, for integers $j, k \geq 0$, repeated differentiation gives that, for some real numbers $\alpha_{1}, \ldots, \alpha_{k}$,

$$
\frac{\partial^{k}}{\partial x^{k}} \mathfrak{E}_{s}^{(j)} h(x)=\sum_{\ell=1}^{k} \frac{\alpha_{\ell}}{x^{k}}\left(\frac{-1}{\sqrt{s} \sigma}\right)^{\ell} \mathfrak{E}_{s}^{(j+\ell)} h(x) .
$$

Note that equation (4.1) says that for $j \geq 1$,

$$
\mathfrak{E}_{s}^{(j)} h(x)=-\sqrt{s} \sigma e^{-r s} \int_{-\infty}^{\infty}\left(x \zeta_{s}(z)\right) h^{\prime}\left(x \zeta_{s}(z)\right) \phi^{(j-1)}(z) d z
$$

In other words

$$
\mathfrak{E}_{s}^{(j)} h(x)=-\sqrt{s} \sigma \mathfrak{E}_{s}^{(j-1)}\left(I h^{\prime}\right)(x) .
$$

Hence if $h \in C^{(1)} \cap \mathcal{K}^{(2)}$, then, for $j \geq 2$, the relation (4.2) can be used a second time, giving

$$
\begin{aligned}
\mathfrak{E}_{s}^{(j-1)}\left(I h^{\prime}\right)(x) & =-\sqrt{s} \sigma \mathfrak{E}_{s}^{(j-2)}\left(I\left(I h^{\prime}\right)^{\prime}\right)(x), \\
\mathfrak{E}_{s}^{(j)} h(x) & =(\sqrt{s} \sigma)^{2}\left(\mathfrak{E}_{s}^{(j-2)}\left(I h^{\prime}\right)(x)+\mathfrak{E}_{s}^{(j-2)}\left(I^{2} h^{\prime \prime}\right)(x)\right) .
\end{aligned}
$$

Noting that

$$
\mathcal{E}_{s} h(x)=\mathfrak{E}_{s}^{(0)} h(x),
$$

we have essentially obtained the following result, which can be used to obtain explicit expressions for $\frac{\partial^{k}}{\partial x^{k}} \mathcal{E}_{s} h(x)$ and $\frac{\partial^{\ell}}{\partial x^{\ell}} \mathcal{E}_{s}^{\langle k\rangle} h(x)$, for any value of $k, \ell \geq 0$.

Theorem 5 (European option derivative representation formulae). For every continuous function $h$ in $\mathcal{K}^{(1)}$, and every pair of integers $j, k \geq 0$, there exist real numbers $\alpha_{1}, \ldots, \alpha_{k}$, such that

$$
x^{k} \frac{\partial^{k}}{\partial x^{k}} \mathfrak{E}_{s}^{(j)} h(x)=\sum_{\ell=1}^{k} \alpha_{\ell} \sqrt{s}^{-\ell} \mathfrak{E}_{s}^{(j+\ell)} h(x) .
$$

When $k \geq 1$, there exist real numbers $\alpha_{1}, \ldots, \alpha_{k}$, such that

$$
x^{k} \frac{\partial^{k}}{\partial x^{k}} \mathfrak{E}_{s}^{(j)} h(x)=\sum_{\ell=0}^{k-1} \alpha_{\ell} \sqrt{s}^{-\ell} \mathfrak{E}_{s}^{(j+\ell)}\left(I h^{\prime}\right)(x) .
$$

When $h \in C^{(1)} \cap \mathcal{K}^{(2)}$ and $k \geq 2$, there exist real numbers $\alpha_{1}, \ldots, \alpha_{k}$ and $\beta_{1}, \ldots, \beta_{k}$, such that

$$
x^{k} \frac{\partial^{k}}{\partial x^{k}} \mathfrak{E}_{s}^{(j)} h(x)=\sum_{\ell=0}^{k-2} \alpha_{\ell} \sqrt{s}^{-\ell} \mathfrak{E}_{s}^{(j+\ell)}\left(I^{2} h^{\prime \prime}\right)(x)+\sum_{\ell=0}^{k-2} \beta_{\ell} \sqrt{s}^{-\ell} \mathfrak{E}_{s}^{(j+\ell)}\left(I h^{\prime}\right)(x) .
$$


In particular, with $h \in C^{(1)} \cap \mathcal{K}^{(2)}$, the following formulae hold:

$$
\begin{aligned}
& x^{2} \mathcal{E}_{s}^{(2)} h(x)=\mathfrak{E}_{s}^{(0)}\left(I^{2} h^{\prime \prime}\right)(x) \\
& x^{3} \mathcal{E}_{s}^{(3)} h(x)=-2 \mathfrak{E}_{s}^{(0)}\left(I^{2} h^{\prime \prime}\right)(x)-\frac{1}{\sigma \sqrt{s}} \mathfrak{E}_{s}^{(1)}\left(I^{2} h^{\prime \prime}\right)(x) \\
& x^{4} \mathcal{E}_{s}^{(4)} h(x)=6 \mathfrak{E}_{s}^{(0)}\left(I^{2} h^{\prime \prime}\right)(x)+\frac{5}{\sqrt{s} \sigma} \mathfrak{E}_{s}^{(1)}\left(I^{2} h^{\prime \prime}\right)(x)+\frac{1}{s \sigma^{2}} \mathfrak{E}_{s}^{(2)}\left(I^{2} h^{\prime \prime}\right)(x)
\end{aligned}
$$

Proof. Equations (4.4), (4.5) and (4.6) follow from the content of the short discussion at the beginning of this section. In order to get expressions for $\frac{\partial^{k}}{\partial x^{k}} \mathcal{E}_{s} h(x)=\frac{\partial^{k}}{\partial x^{k}} \mathfrak{E}_{s}^{(0)}(h)(x), k=2,3,4$, one first calculates the actual values of $\alpha_{j}, j=0, \ldots, 4$, such that (4.4) holds, and repeatedly uses (4.2) and (4.3). This is tedious but otherwise trivial.

Remark 5 (Expressing $\mathfrak{E}_{s}^{(\ell)}$ in terms of $\mathcal{E}_{s}$ ). Recall $\eta_{s}$ from (1.5) and note that $\eta_{s}\left(\zeta_{s}(z)\right)=z$, and $\frac{\phi^{(\ell)}(z)}{\phi(z)}$ is a polynomial in $z$. Expressions involving $\mathfrak{E}_{s}^{(\ell)}\left(I^{2} h^{\prime \prime}\right)(x)$ can also be written in terms of $\mathcal{E}_{s}$ in the following manner:

$$
\begin{aligned}
\mathfrak{E}_{s}^{(\ell)}\left(I^{2} h^{\prime \prime}\right)(x) & =e^{-r s} \int_{-\infty}^{\infty}\left(x \zeta_{s}(z)\right)^{2} h^{\prime \prime}\left(x \zeta_{s}(z)\right) \frac{\phi^{(\ell)}\left(\eta_{s}\left(\frac{x \zeta_{s}(z)}{x}\right)\right)}{\phi\left(\eta_{s}\left(\frac{x \zeta_{s}(z)}{x}\right)\right)} \phi(z) d z \\
& =\mathcal{E}_{s}\left(I^{2} h^{\prime \prime} \frac{\phi^{(\ell)}\left(\eta_{s}\left(\frac{I}{x}\right)\right)}{\phi\left(\eta_{s}\left(\frac{I}{x}\right)\right)}\right)(x) \\
& =e^{-r s} E_{x}\left(\xi_{s}^{2} h^{\prime \prime}\left(\xi_{s}\right) \frac{\phi^{(\ell)}\left(\eta_{s}\left(\frac{\xi_{s}}{x}\right)\right)}{\phi\left(\eta_{s}\left(\frac{\xi_{s}}{x}\right)\right)}\right) .
\end{aligned}
$$

In the statement of Theorem 2, we use the last form of this equation.

Remark 6 (Boundedness of $\mathcal{E}_{s}^{\langle k\rangle} g$ and $x^{m} \frac{\partial^{m}}{\partial x^{m}} \mathcal{E}_{s}^{\langle k\rangle} g$ ). With the European option derivative representation formulae, functions of the form $I^{\ell} \frac{\partial^{\ell}}{\partial x^{\ell}} \mathcal{E}_{t_{j}}^{\langle k\rangle} g$ are easy to deal with. If for instance $k, \ell \geq 0$ and $k+\ell \geq 2$ and $g \in C^{(1)} \cap \mathcal{K}^{(2)}$, then

$$
\begin{aligned}
I^{\ell} \frac{\partial^{\ell}}{\partial x^{\ell}} \mathcal{E}_{t_{j}}^{\langle k\rangle} g & =\sum_{j=0}^{k+\ell-2} \alpha_{j} \sqrt{s}^{-j} \mathfrak{E}_{s}^{(j)}\left(I^{2} g^{\prime \prime}\right)(x) \\
& +\sum_{j=0}^{k+\ell-2} \beta_{j} \sqrt{s}^{-j} \mathfrak{E}_{s}^{(j)}\left(I g^{\prime}\right)(x),
\end{aligned}
$$

for some real numbers $\alpha_{i}, \beta_{i}, i=0, \ldots, k+\ell-2$. The fact that such expressions are polynomially bounded immediately follows from the fact that, given real numbers $a, b \geq 0$ and an integer $j \geq 0$, there exists a constant $Q$, such that for every function $\psi$ satisfying $|\psi(x)| \leq a\left(1+x^{b}\right)$, and every $x, s>0$,

$$
\left|\mathfrak{E}_{s}^{(j)}(\psi)(x)\right| \leq Q a\left(1+x^{b}\right) .
$$




\section{The PAYOFF SMOOTHING ERROR}

Recall $\chi_{p}(h)$ from (1.8).

Theorem 6. For every $h \in C^{(1)} \cap \mathcal{K}_{p}^{(3)}$ and every $x>0$,

$$
\operatorname{Err}_{T}^{n}\left(h-\mathcal{E}_{\frac{T}{n}}(h)\right)(x)=\chi_{p}(h)\left(1+x^{p+3}\right) \mathcal{O}\left(n^{-1.5}\right) .
$$

Proof. We get from the Taylor expansion theorem that

$$
\begin{aligned}
\mathcal{E}_{\frac{T}{n}} h(x) & =e^{-r \frac{T}{n}} h(x)+x h^{\prime}(x) \mathcal{E}_{\frac{T}{n}}(I-1) \\
& +\mathcal{E}_{\frac{T}{n}}\left(\int_{1}^{I}(x u)^{2} h^{\prime \prime}(x u) \frac{(I-u)}{u^{2}} d u\right)(1) .
\end{aligned}
$$

Hence, since from risk neutrality, $\mathcal{E}_{\frac{T}{n}}(I-1)=1-e^{-r \frac{T}{n}}=\mathcal{O}\left(n^{-1}\right)$,

$$
\begin{aligned}
h(x)-\mathcal{E}_{\frac{T}{n}} h(x) & =\left(1-e^{-r \frac{T}{n}}\right)\left(h(x)-x h^{\prime}(x)\right) \\
& -\mathcal{E}_{\frac{T}{n}}\left(\int_{1}^{I}(x u)^{2} h^{\prime \prime}(x u) \frac{(I-u)}{u^{2}} d u\right)(1),
\end{aligned}
$$

and therefore

$$
\begin{aligned}
\operatorname{Err}_{T}^{n}\left(h-\mathcal{E}_{\frac{T}{n}}(h)\right)(x) & =\mathcal{O}\left(n^{-1}\right) \operatorname{Err}_{T}^{n}\left(h-I h^{\prime}\right)(x) \\
& -\mathcal{E}_{\frac{T}{n}}\left(\int_{1}^{I}\left(\operatorname{Err}_{T}^{n}\left(I^{2} h^{\prime \prime}\right)(x u)\right) \frac{(I-u)}{u^{2}} d u\right)(1) .
\end{aligned}
$$

But $h-I h^{\prime} \in C^{(0)} \cap \mathcal{K}_{p+2}^{(2)}$, and $I^{2} h^{\prime \prime} \in \mathcal{K}_{p+2}^{(1)}$. Hence, from Proposition 2 below,

$$
\begin{aligned}
\left|\operatorname{Err}_{T}^{n}\left(h-I h^{\prime}\right)(x)\right| & \leq \mathcal{O}\left(n^{-0.5}\right) \chi_{p}(h)\left(1+(x)^{p+3}\right) \\
\left|\operatorname{Err}_{T}^{n}\left(I^{2} h^{\prime \prime}\right)(x u)\right| & \leq \mathcal{O}\left(n^{-0.5}\right) \chi_{p}(h)\left(1+(x u)^{p+3}\right)
\end{aligned}
$$

Now

$$
\left|\int_{1}^{I}\left(1+(x u)^{p+3}\right) \frac{(I-u)}{u^{2}} d u\right|=\int_{1}^{I}\left(1+(x u)^{p+3}\right) \frac{(I-u)}{u^{2}} d u,
$$

and since, by $\mathrm{P} 5$,

$$
\mathcal{E}_{\frac{T}{n}}\left(\int_{1}^{I}\left(1+(x u)^{p+3}\right) \frac{(I-u)}{u^{2}} d u\right)=\mathcal{O}\left(n^{-1}\right)\left(1+x^{p+3}\right),
$$

we obtain

$$
\mathcal{E}_{\frac{T}{n}}\left(\int_{1}^{I} \operatorname{Err}_{T}^{n}\left(I^{2} h^{\prime \prime}\right)(x u) \frac{(I-u)}{u^{2}} d u\right)(1)=\mathcal{O}\left(n^{-\frac{3}{2}}\right) \chi_{p}(h)\left(1+x^{p+3}\right) .
$$




\section{Auxiliary Results}

We list here basic properties satisfied by all flexible CRR Schemes. Here $F_{\xi_{t}^{(n)}}$ and $F_{\xi_{t}}$ denote the cumulative distribution functions of $\xi_{t}^{(n)}$ and $\xi_{t}$, with $\xi_{0}^{(n)}=\xi_{0}$. Proofs are left to the reader.

Lemma 2 (Properties of $\left\{\xi^{(n)}\right\}$ ). Every flexible CRR Scheme $\left\{\xi^{(n)}\right\}_{n=1}^{\infty}$ satisfies the following properties:

P1 (Berry-Esseen): There exists a constant $Q$ such that for every $t \in \frac{T}{n} \mathbb{N}$, with $\frac{T}{2} \leq t \leq T$,

$$
\sup _{z}\left|F_{\xi_{t}^{(n)}}(z)-F_{\xi_{t}}(z)\right| \leq Q n^{-\frac{1}{2}}
$$

P2 (local estimate of the distance to 1$)$ : For integers $k \geq 0$,

$$
\delta_{k}^{(n)}:=\mathcal{E}_{\frac{T}{n}}^{n}\left(|I-1|^{k}\right)(1)=\mathcal{O}\left(n^{-\frac{k}{2}}\right) .
$$

P3 (local error of the difference from 1): For integers $k=2,3,4$, there exist $\Delta_{k}$ such that

$$
\Delta_{k}^{(n)}:=\operatorname{Err}_{\frac{T}{n}}^{n}\left((I-1)^{k}\right)(1)=\frac{\Delta_{k}}{n^{2}}+\mathcal{O}\left(n^{-\frac{5}{2}}\right) .
$$

P4 (local and global estimates for $\log$ and power functions):

$$
\mathcal{E}_{\frac{T}{n}}^{n}(|\ln (I)|)(1)=\mathcal{O}\left(n^{-\frac{1}{2}}\right) .
$$

Furthermore, for every fixed real number $\gamma$,

$$
\mathcal{E}_{\frac{T}{n}}^{n}\left(I^{\gamma}\right)=\mathcal{E}_{\frac{T}{n}}\left(I^{\gamma}\right)+\mathcal{O}\left(n^{-2}\right)
$$

and (consequently)

$$
\begin{aligned}
\mathcal{E}_{\frac{T}{n}}^{n}\left(\left|I^{\gamma}-1\right|\right)(1) & =\mathcal{O}\left(n^{-\frac{1}{2}}\right), \\
\max _{j=0, \ldots, n}\left|\mathcal{E}_{\frac{j T}{n}}^{n}\left(I^{\gamma}\right)(x)-\mathcal{E}_{\frac{j T}{n}}\left(I^{\gamma}\right)(x)\right| & =x^{\gamma} \mathcal{O}\left(n^{-1}\right), \\
\max _{j=0, \ldots, n}\left|\mathcal{E}_{\frac{j T}{n}}^{n}\left(I^{\gamma}\right)(x)\right| & =x^{\gamma} \mathcal{O}(1) .
\end{aligned}
$$

P5 (Remainder related local estimate): For any integer $\beta$ and any integer $N \geq 0$,

$$
\mathcal{E}_{\frac{T}{n}}^{n}\left(\left|\int_{1}^{I} u^{\beta}(I-u)^{N} d u\right|\right)(1)=\mathcal{O}\left(n^{-\frac{N+1}{2}}\right) .
$$

Remark 7. All the properties P1-P5 remain valid if $\xi^{(n)}$ is replaced by $\xi$.

Recall the notation of section 4 . The following lemma is a practical and simple result. 
Lemma 3. Let integer $k \geq 0$. For every $h \in C^{(k-1)} \cap \mathcal{K}^{(k)}, s>0$ and $x \geq 0$,

$$
x^{k} \frac{d^{k}}{d x^{k}} \mathcal{E}_{s} h(x)=\mathcal{E}_{s}\left(I^{k} \frac{d^{k}}{d x^{k}} h\right)(x) .
$$

Proof. Since $\mathcal{E}_{s} h(x)=e^{-r s} \int_{-\infty}^{\infty} h\left(x \zeta_{s}(z)\right) \phi(z) d z$, one writes

$$
\begin{aligned}
x^{k}\left(\mathcal{E}_{s} h\right)^{(k)}(x) & =x^{k} e^{-r s} \frac{d^{k}}{d x^{k}} \int_{-\infty}^{\infty} h\left(x \zeta_{s}(z)\right) \phi(z) d z \\
& =x^{k} e^{-r s} \int_{-\infty}^{\infty}\left(\zeta_{s}(z)\right)^{k} h^{(k)}\left(x \zeta_{s}(z)\right) \phi(z) d z \\
& =e^{-r s} \int_{-\infty}^{\infty}\left(x \zeta_{s}(z)\right)^{k} h^{(k)}\left(x \zeta_{s}(z)\right) \phi(z) d z \\
& =\mathcal{E}_{s}\left(I^{k} h^{(k)}\right)(x) .
\end{aligned}
$$

The result below extends the Berry-Esseen property P1.

Proposition 2. If $\varphi \in \mathcal{K}_{p}^{(1)}$ then,

$$
\max _{\frac{T}{2} \leq t_{m} \leq T}\left|\operatorname{Err}_{t_{m}}^{n} \varphi(x)\right| \leq\left(\|\varphi\|_{p}^{(1)}+\sum|\Delta \varphi|\right)\left(1+x^{p+1}\right) \mathcal{O}\left(n^{-\frac{1}{2}}\right) .
$$

Proof. If $\varphi$ belongs to $\mathcal{K}_{p}^{(1)}$, but is not continuous, then it can be decomposed into a (finite) sum of piecewise constant functions and a continuous function $\varphi_{*}$ in $\mathcal{K}_{p}^{(1)}$. Note that $\left\|\varphi_{*}\right\|_{p}^{(1)} \leq\|\varphi\|_{p}^{(1)}+\sum|\Delta \varphi|$. Since the convergence of option values occurs at a rate of a least $n^{-\frac{1}{2}}$ when the payoff is piecewise constant, due to property $\mathrm{P} 1$ in section 6 , we can assume, without loss of generality, that $\varphi \in C^{(0)} \cap \mathcal{K}_{p}^{(1)}$. Recall that, due to the European option derivative representation formulae, there exists a constant $Q$ such that, for $0<s \leq T$ and for $k=2, \ldots, 5$,

$$
\left|\mathcal{E}_{s}^{\langle k\rangle}(\varphi)(x)\right| \leq Q \frac{\|\varphi\|_{p}^{(1)}}{\sqrt{s}^{k-1}}\left(1+x^{p+1}\right) .
$$

Let $t_{m}$ be a time step in the interval $\left[\frac{T}{2}, T\right]$. Substituting the above estimate in the error localization expansion formula (with $M=0$ and $N=4$ ) we get, invoking Remark 3 and property P3 in section 6,

$$
\begin{aligned}
\operatorname{MErr}_{t_{m}}^{n} \varphi(x) & =m \sum_{k=2}^{4} \frac{\mathcal{O}\left(n^{-2}\right)}{k !} \frac{\|\varphi\|_{p}^{(1)}}{{\sqrt{t_{m}}}^{k-1}}\left(1+x^{p+1}\right) \\
& +m \frac{\|\varphi\|_{p}^{(1)}}{{\sqrt{t_{m}}}^{k-1}}\left(1+x^{p+1}\right) \mathcal{O}\left(n^{-\frac{5}{2}}\right) \\
& =\|\varphi\|_{p}^{(1)}\left(1+x^{p+1}\right) \mathcal{O}\left(n^{-1}\right)
\end{aligned}
$$


Using additionally the fact that $\operatorname{Err}_{t_{\ell}}^{n}$ and $\mathcal{R}_{\frac{T}{n}}^{n, N}$ commute, we similarly get

$$
\begin{aligned}
\operatorname{CErr}_{t_{m}}^{n} \varphi(x) & =\sum_{j=1}^{m-1} \sum_{k=2}^{4} \frac{\mathcal{O}\left(n^{-2}\right)}{k !} \frac{\|\varphi\|_{p}^{(1)}}{{\sqrt{t_{j}}}^{k-1}}\left(1+x^{p+1}\right) \\
& +\sum_{j=1}^{m-1} \frac{\|\varphi\|_{p}^{(1)}}{{\sqrt{t_{j}}}^{4}}\left(1+x^{p+1}\right) \mathcal{O}\left(n^{-\frac{5}{2}}\right) \\
& +\|\varphi\|_{p}^{(1)}\left(1+x^{p+1}\right) \mathcal{O}\left(n^{-\frac{1}{2}}\right) \\
& =\|\varphi\|_{p}^{(1)}\left(1+x^{p+1}\right) \mathcal{O}\left(n^{-\frac{1}{2}}\right) .
\end{aligned}
$$

The result below is used in the proof of Theorem 4 .

Lemma 4. Let $k \geq 2$ be an integer and $g \in C^{(1)} \cap \mathcal{K}_{p}^{(3)}$. Then,

$$
\left|\operatorname{Err}_{T-t_{m}}^{n}\left(\mathcal{E}_{t_{m}}^{\langle k\rangle} g\right)(x)\right| \leq \frac{\chi_{p}(g)}{{\sqrt{t_{m}}}^{k-1}} \mathcal{O}\left(n^{-1}\right)\left(1+x^{p+3}\right),
$$

for all time steps $0<t_{m}<T$ and $x \geq 0$.

Proof. Note that, by Lemma 3, for every $s, t \geq 0$,

$$
\mathcal{E}_{s} \mathcal{E}_{t}^{\langle k\rangle} g(x)=\mathcal{E}_{s}^{\langle k\rangle} \mathcal{E}_{t} g(x)=\mathcal{E}_{s+t}^{\langle k\rangle} g(x) .
$$

The error localization expansion formula (with $M=N=4$ ) gives

$$
\begin{aligned}
\operatorname{MErr}_{T-t_{m}}^{n} \mathcal{E}_{t_{m}}^{\langle k\rangle} g(x) & =m \sum_{\ell=2}^{4} \frac{\Delta_{\ell}^{(n)}}{\ell !} x^{\ell} \frac{\partial^{\ell}}{\partial x^{\ell}} \mathcal{E}_{T-t_{1}}^{\langle k\rangle} g(x) \\
& +m \mathcal{R}_{\frac{T}{n}}^{n, 4}\left(I^{5} \frac{\partial^{5}}{\partial x^{5}} \mathcal{E}_{T-t_{1}}^{\langle k\rangle} g\right)(x)
\end{aligned}
$$

$$
\begin{aligned}
\operatorname{CErr}_{T-t_{m}}^{n} \mathcal{E}_{t_{m}}^{\langle k\rangle} g(x) & =\sum_{j=0}^{m-1} \sum_{\ell=2}^{4} \frac{\Delta_{\ell}^{(n)}}{\ell !} \operatorname{Err}_{T-t_{m}-t_{j+1}}^{n}\left(x^{\ell} \frac{\partial^{\ell}}{\partial x^{\ell}} \mathcal{E}_{t_{j}+t_{m}}^{\langle k\rangle} g\right)(x) \\
& +\sum_{j=0}^{m-1} \mathcal{R}_{\frac{T}{n}}^{n, 4}\left(\operatorname{Err}_{T-t_{m}-t_{j+1}}^{n}\left(x^{5} \frac{\partial^{5}}{\partial x^{5}} \mathcal{E}_{t_{j}+t_{m}}^{\langle k\rangle} g\right)\right)(x)
\end{aligned}
$$

By the European option derivative representation formulae, there exists a constant $Q$, such that for $k, \ell=2, \ldots, 5$,

$$
\left|x^{\ell} \frac{\partial^{\ell}}{\partial x^{\ell}} \mathcal{E}_{s}^{\langle k\rangle} g\right| \leq Q \frac{\|g\|_{p}^{(2)}}{\sqrt{s}^{(k+\ell-2)}}\left(1+x^{p+1}\right) .
$$

Using Remark 3 and property P3 in section 6, replacing this estimate in the above formulae one gets not only that

$$
\max _{0<t_{m}<T}\left|\operatorname{MErr}_{T-t_{m}}^{n} \mathcal{E}_{t_{m}}^{\langle k\rangle} g(x)\right|=\|g\|_{p}^{(2)}\left(1+x^{p+2}\right) \mathcal{O}\left(n^{-1}\right),
$$


but also, that the contribution in $\left|\operatorname{CErr}_{T-t_{m}}^{n} \mathcal{E}_{t_{m}}^{\langle k\rangle} g(x)\right|$ of those $t_{j}$ 's for which

$$
\frac{T}{2} \leq t_{j}+t_{m} \leq T
$$

amounts to $\|g\|_{p}^{(2)}\left(1+x^{p+2}\right) \mathcal{O}\left(n^{-1}\right)$. Hence we are left to consider the $t_{j}$ 's for which $0<t_{j}+t_{m}<\frac{T}{2}$, in which case

$$
\frac{T}{2} \leq T-t_{m}-t_{j+1}<T
$$

Let us write $s:=s(j, m, n)=t_{j}+t_{m}$ and $t:=t(j, m, n)=T-t_{m}-$ $t_{j+1}$. Recall that, by the European option derivative representation formulae, $x^{\ell} \frac{\partial^{\ell}}{\partial x^{\ell}} \mathcal{E}_{s}^{\langle k\rangle} g(x)$ is a linear combination of terms of the form $s^{-\frac{j}{2}} \mathfrak{E}_{s}^{(j)}\left(I g^{\prime}\right)(x)$ and $s^{-\frac{j}{2} \mathfrak{E}_{s}^{(j)}}\left(I^{2} g^{\prime \prime}\right)(x)$, with $j \in\{0, . ., k+\ell-2\}$. Now

$$
\mathfrak{E}_{s}^{(j)}\left(I^{2} g^{\prime \prime}\right)(x)=\mathcal{E}_{s}\left((x I)^{2} g^{\prime \prime}(x I) \frac{\phi^{(j)}\left(\eta_{s}(I)\right)}{\phi\left(\eta_{s}(I)\right)}\right)(1),
$$

so that

$$
\operatorname{Err}_{t}^{n}\left(\mathfrak{E}_{s}^{(j)}\left(I^{2} g^{\prime \prime}\right)\right)(x)=\mathfrak{E}_{s}^{(j)}\left(\operatorname{Err}_{t}^{n}\left(I^{2} g^{\prime \prime}\right)\right)(x),
$$

and (recall that $\frac{T}{2} \leq t<T$ ) our extension to the Berry-Esseen theorem, Proposition 2, yields

$$
\left|\operatorname{Err}_{t}^{n}\left(I^{2} g^{\prime \prime}\right)(x)\right|=\chi_{p}(g) \mathcal{O}\left(n^{-\frac{1}{2}}\right)\left(1+x^{p+3}\right) .
$$

Since the same is true for the terms $\mathfrak{E}_{s}^{(j)}\left(I g^{\prime}\right)(x)$, it follows that

$$
\operatorname{Err}_{t}^{n}\left(I^{\ell} \frac{\partial^{\ell}}{\partial x^{\ell}} \mathcal{E}_{s}^{\langle k\rangle} g\right)(x)=\sqrt{s}^{-(k+\ell-2)} \chi_{p}(g) \mathcal{O}\left(n^{-\frac{1}{2}}\right)\left(1+x^{p+3}\right) .
$$

Noticing that, with constant $\mathcal{Q}:=2(1+T)^{4}$,

$$
\|g\|_{p}^{(2)}\left(1+x^{p+2}\right) \leq \mathcal{Q} \sqrt{s}^{-(k+\ell-2)} \chi_{p}(g)\left(1+x^{p+3}\right)
$$

for every $s, x>0$, and $k, \ell=2, \ldots, 5$, we can use the estimate (6.2) in formula (6.1), to obtain

$$
\begin{aligned}
& \left|\operatorname{CErr}_{T-t_{m}}^{n} \mathcal{E}_{t_{m}}^{\langle k\rangle} g(x)\right| \\
& =\sum_{j=0}^{n-m-1} \sum_{\ell=2}^{4}\left(\frac{\mathcal{O}\left(n^{-2.5}\right)}{{\sqrt{t_{m}+t_{j}}}^{k+\ell-2}}+\frac{\mathcal{O}\left(n^{-3}\right)}{{\sqrt{t_{m}+t_{j}}}^{k+3}}\right) \chi_{p}(g)\left(1+x^{p+3}\right) .
\end{aligned}
$$

From this one easily gets

$$
\left|\mathrm{CErr}_{T-t_{m}}^{n} \mathcal{E}_{t_{m}}^{\langle k\rangle} g(x)\right|=\frac{\mathcal{O}\left(n^{-1}\right)}{{\sqrt{t_{m}}}^{k-1}} \chi_{p}(g)\left(1+x^{p+3}\right) .
$$




\section{REFERENCES}

[1] Carbone, R., Binomial approximation of Brownian motion and its maximum, Statistics and Probability Letters 69 no. 3, 271-285 (2004). doi:10.1016/j.spl.2004.06.020

[2] Chang, L.B. and Palmer, K., Smooth convergence in the binomial model, Finance and Stochastics 11 no. 1, 91-105 (2007). doi:10.1007/s00780-006-0020-6

[3] Cox, J.C., Ross, S.A. and Rubinstein, M., Option pricing: a simplified approach, Journal of Financial Economics 7, 229-263 (1979). doi:10.1016/0304-405X(79)900151

[4] Diener, F. and Diener, M., Asymptotics of the price oscillations of a European call option in a tree model, Mathematical finance 14 no. 2, 271-293 (2004).doi:10.1111/j.0960-1627.2004.00192.x

[5] Diener, F. and Diener, M., Higher-order terms for the de Moivre-Laplace theorem, Contemporary Mathematics 373 191-206 (2005). doi:10.1090/conm/373

[6] Dupuis, P. and Wang, H., Optimal stopping with random intervention times, Advances in Applied probability 34 no. 1, 141-157 (2002). doi:10.1239/aap/1019160954

[7] Dupuis, P. and Wang, H., On the convergence from discrete to continuous time in an optimal stopping problem, Annals of Applied Probability 1339-1366 (2005). doi:10.1214/105051605000000034

[8] Heston, S. and Zhou, G., On the rate of convergence of discrete-time contingent claims, Mathematical Finance 10 no. 1, 53-75 (2000). doi:10.1111/1467-9965.00080

[9] Hu, B., Liang, J. and Jiang, L., Optimal convergence rate of the explicit finite difference scheme for American option valuation, Journal of Computational and Applied Mathematics 230 no. 2, 583-599 (2009). doi:10.1016/j.cam.2008.12.018

[10] Joshi, M.S., Achieving smooth asymptotics for the prices of European options in binomial trees, Quantitative Finance 9 no. 2, 171-176 (2009). doi:10.1080/14697680802624955

[11] Joshi, M.S., Achieving higher order convergence for the prices of European options in binomial trees, Mathematical Finance 20 no. 1, 89-103 (2010). doi:10.1111/j.14679965.2009.00390.x

[12] Kifer, Y., Error estimates for binomial approximations of game options, Annals of Applied Probability 16 no. 2, 984-1033 (2006). doi:10.1214/105051606000000808

[13] Korn, R., and Müller, S., The optimal-drift model: an accelerated binomial scheme, Finance and Stochastics, 1-26 (2012). doi:10.1007/s00780-012-0179-y

[14] Lamberton, D., Error estimates for the binomial approximation of American put options, The Annals of Applied Probability 8 no. 1, 206-233 (1998). doi:10.1214/aoap/1027961041

[15] Lamberton, D., Vitesse de convergence pour des approximations de type binomial, Ecole CEA, EDF, INRIA mathématiques financières: modèles économiques et mathématiques des produits dérivés 347-359 (1999).

[16] Lamberton, D., Brownian optimal stopping and random walks, Applied Mathematics and Optimization 45 no. 3, 283-324 (2002). doi:10.1007/s00245-001-0033-7

[17] Lamberton, D. and Rogers, LCG, Optimal stopping and embedding, Journal of Applied Probability 37 no. 4, 1143-1148 (2000). doi:10.1239/jap/1014843094

[18] Leduc, G., Exercisability Randomization of the American Option, Stochastic Analysis and Applications 26 no. 4, 832-855 (2008). doi:10.1080/07362990802128669

[19] Leduc, G., Convergence rate of the binomial tree scheme for continuously paying options, Annales des sciences mathématiques du Québec 36 no. 2, 381-394 (2012).

[20] Leisen, D.P.J., Pricing the American put option: A detailed convergence analysis for binomial models, Journal of Economic Dynamics and Control 22 no. 8-9, 1419-1444 (1998). doi:10.1016/S0165-1889(98)00019-0 
[21] Leisen, D.P.J. and Reimer, M., Binomial models for option valuation-examining and improving convergence, Applied Mathematical Finance 3 no. 4, 319-346 (1996). doi:10.1080/13504869600000015

[22] Liang, J., Hu, B., Jiang, L. and Bian, B., On the rate of convergence of the binomial tree scheme for American options, Numerische Mathematik 107 no. 2, 333-352 (2007). doi:10.1007/s00211-007-0091-0

[23] Lin, J. and Palmer, K., Convergence of barrier option prices in the binomial model, Mathematical Finance 23 no. 2, 318-338 (2013). doi:10.1111/j.14679965.2011.00501.x

[24] Tian, Y., A flexible binomial option pricing model, Journal of Futures Markets 19 no. 7, 817-843 (1999). doi:10.1002/(SICI)1096-9934(199910)19:7<817::AIDFUT5 $>3.0 . \mathrm{CO} ; 2-\mathrm{D}$

[25] Walsh, J.B., The rate of convergence of the binomial tree scheme, Finance and Stochastics 7 no. 3, 337-361 (2003). doi:10.1007/s007800200094

American University of Sharjah, P.O. Box 26666, Sharjah, United Arab EMIRATES

Email address: gleduc@aus.edu 